\title{
JOINT NUMERICAL RANGES AND COMPRESSIONS OF POWERS OF OPERATORS
}

\author{
VLADIMIR MÜLLER AND YURI TOMILOV
}

\begin{abstract}
We identify subsets of the joint numerical range of an operator tuple in terms of its joint spectrum. This result helps us to transfer weak convergence of operator orbits into certain approximation and interpolation properties for powers in the uniform operator topology. This is a far-reaching generalization of one of the main results in our recent paper [26]. Moreover, it yields an essential (but partial) generalization of Bourin's "pinching" theorem from [7. It also allows us to revisit several basic results on joint numerical ranges, provide them with new proofs and find a number of new results.
\end{abstract}

\section{INTRODUCTION}

The theory of joint numerical ranges is a developing area of operator theory with several important results obtained in the last years. The geometric structure of joint numerical ranges has got a considerable attention, and many properties of numerical ranges have been transferred or appropriately recasted from the setting of a single operator to the framework of operator tuples, see e.g. [3, [19]-[22], 25], 26] and references therein. At the same time, the relations between spectrum of an operator tuple and its numerical range remained rather obscure until very recent time. We are aware of [33] as the only important contribution to those issues, which moreover was apparently overlooked by the experts. Recently in [26], we have discovered new spectral inclusion results for operator tuples. Specified for tuples formed by powers of a single operator, the results allowed us to identify the unit circle in the spectrum of a bounded linear operator on a Hilbert space in terms of orthogonality and "quasi-orthogonality" relations for the operator orbits, see e.g. [26, Theorem 1.1]. This constituted an essential generalization of the corresponding results by Arveson [2], who dealt with unitary operators only. Moreover, in [26], by means of spectral approximations of numerical ranges, we put recent harmonic analysis considerations by Hamdan ([16]) into the operator setting and extended them by, in particular, replacing a

1991 Mathematics Subject Classification. Primary 47A05, 47A10, 47A12; Secondary 47A30, 47A35, 47D03.

This work was partially supported by the NCN grant 2014/13/B/ST1/03153, by the EU grant "AOS", FP7-PEOPLE-2012-IRSES, No 318910, by grant No. 17-27844S of GA CR and RVO:67985840. 
single orbit in Hamdan's statement by an infinite-dimensional subspace of its orbits. (See below for more on that.)

The present paper brings further insights into relations between spectrum and numerical range for operator tuples, and uses them to obtain new asymptotic properties of operator orbits under quite general assumptions. More precisely, we extend, complement and sharpen several main results from [26] on numerical ranges $W\left(T_{1}, \ldots, T_{n}\right)$ of tuples $\mathcal{T}=\left(T_{1}, \ldots, T_{n}\right)$ of bounded linear operators on a Hilbert space $H$, and in this way obtain essential generalizations of results from [26] on asymptotic behavior of compressions of operator powers. As a consequence, we obtain a partial generalization of the "pinching" theorem by Bourin [7, Theorem 2.1] in a much more demanding setting of operator tuples. For recent applications of [7, Theorem 2.1] see [8].

One of the novelties in our approach, stemming from [26], is that in our studies of geometric properties of operator iterates we rely on the numerical ranges methodology. It is instructive to note that the condition of orthogonality of elements from an orbit of $T \in B(H)$ can be rewritten in terms of the joint numerical range of the tuple $\mathcal{T}=\left(T, \ldots, T^{n}\right)$. On the other hand, as we prove below, the joint numerical range $W(\mathcal{T})$ contains the interior of the convex hull of the joint spectrum $\sigma(\mathcal{T}$ ) (in spite of the fact that the joint numerical range is in general not convex). Using inductive arguments, this fact helps us to construct orbits of $T$ with special geometric properties from the vectors resembling (essential) approximate eigenvectors of $T$. The constructions are far from being straightforward, and we have to invoke new ideas not present in [26]. More precisely, our considerations rely on the following spectral inclusion result.

Theorem 1.1. Let $\mathcal{T}=\left(T_{1}, \ldots, T_{n}\right) \in B(H)^{n}$. Then

$$
\text { Int conv }\left(W_{e}(\mathcal{T}) \cup \sigma_{p}(\mathcal{T})\right) \subset W(\mathcal{T}) .
$$

Moreover, if the tuple $\mathcal{T}$ is commuting then

$$
\text { Int conv } \sigma(\mathcal{T}) \subset W(\mathcal{T}) .
$$

Theorem 1.1 can be considered as a partial generalization of the main result in [33, Theorem 2.2] dealing with numerical ranges of operators on Banach spaces, and also as a generalization of [26, Corollaries 4.2 and 4.4], where $\sigma_{p}(\mathcal{T})$ was absent in (1.1), and (1.2) was stated with $\sigma(\mathcal{T})$ replaced by $\sigma_{e}(\mathcal{T})$. Note that while the result in [33] allows to find parts of the spectrum of $\mathcal{T}$ in $\overline{W(\mathcal{T})}$, we may replace $\overline{W(\mathcal{T})}$ by a smaller and more transparent set $W(\mathcal{T})$. The proof of Theorem 1.1 requires new tools, e.g. Zenger's Lemma, and it is technically more demanding than the corresponding arguments in [26].

To present our applications of Theorem 1.1 (or rather its predecessor from [26]), let us recall that, motivated by applications in ergodic theory, Hamdan characterized in [16] the size of the spectrum of some unitary operators by a new type of asymptotic assumptions. He proved that if a unitary operator 
$T$ on $H$ is such that $T^{n} \rightarrow 0$ in the weak operator topology, then $\sigma(T)=\mathbb{T}$ if and only if for every $\epsilon>0$ there exists a unit vector $x \in H$ satisfying

$$
\sup _{n \geq 1}\left|\left\langle T^{n} x, x\right\rangle\right|<\epsilon .
$$

His result has been extended in [26] to general bounded operators and to the setting allowing to take $x$ 's in (1.3) from an infinite-dimensional subspace. Namely, we proved in [26, Corollary 6.3 and Remark 6.4] that if $T$ is a bounded linear operator on $H$ such that $T^{n} \rightarrow 0$ in the weak operator topology, and $\sigma(T) \supset \mathbb{T}$, then for every $\epsilon>0$ one can find an infinitedimensional subspace $L$ of $H$ such that the compressions $\left(T^{n}\right)_{L}$ of $T^{n}$ to $L$ are asymptotically small in two senses:

$$
\lim _{n \rightarrow \infty}\left\|\left(T^{n}\right)_{L}\right\|=0 \quad \text { and } \quad \sup _{n \geq 1}\left\|\left(T^{n}\right)_{L}\right\|<\epsilon .
$$

This result can be generalized as follows.

Theorem 1.2. Let $T \in B(H)$ be such that $T^{n} \rightarrow 0$ in the weak operator topology, and let $\sigma(T) \supset \mathbb{T}$. Let $\tilde{C}$ be a strict contraction on a separable Hilbert space, i.e., $\|\tilde{C}\|<1$. Then for every $\varepsilon>0$ there exists a subspace $L \subset H$ and $C \in B(L)$ unitarily equivalent to $\tilde{C}$ such that

$$
\lim _{n \rightarrow \infty}\left\|\left(T^{n}\right)_{L}-C^{n}\right\|=0 \quad \text { and } \quad \sup _{n \geq 1}\left\|\left(T^{n}\right)_{L}-C^{n}\right\| \leq \varepsilon .
$$

It is natural to ask whether the asymptotic relation (1.4) above can be made an exact equality. Surprisingly, the answer is "yes", if one restricts oneself to a finite piece of the orbit $\left(C^{n}\right)_{n \geq 1}$. In particular, the following theorem holds.

Theorem 1.3. Let $T \in B(H)$ and suppose that the polynomial hull $\hat{\sigma}(T)$ of $\sigma(T)$ contains the unit disc $\mathbb{D}$. Let $n \in \mathbb{N}$ and let $\tilde{C}$ be a strict contraction on a separable Hilbert space. Then there exists a subspace $L \subset H$ and $C \in B(L)$ unitarily equivalent to $\tilde{C}$ such that $\left(T^{k}\right)_{L}=C^{k}$ for $k=1, \ldots, n$.

The question when it is possible to obtain the equality $\left(T^{k}\right)_{L}=C^{k}$ for all $k \in \mathbb{N}$ (i.e., when $T$ is a dilation of $C$ ) was studied by using the Scott Brown technique. In particular, in [4, Theorem 4.8] a positive result was obtained for so called BCP-operators (contractions with dominant essential spectrum).

Despite the main motivation for the paper was to understand how far Hamdan's type results can be pushed by operator-theoretical technique, as a byproduct of our approach we found new arguments for the proofs of recent characterizations of essential and infinite numerical ranges, as well as several new statements concerning numerical ranges which are of independent interest. Recall that the infinite numerical range $W_{\infty}(\mathcal{T})$ of a tuple $\mathcal{T}=\left(T_{1}, \ldots, T_{n}\right)$ can be defined as

$$
W_{\infty}(\mathcal{T}):=\left\{\left(\lambda_{1}, \ldots, \lambda_{n}\right) \in \mathbb{C}^{n}: P T_{j} P=\lambda_{j} P, \quad j=1, \ldots, n\right\}
$$


for some infinite rank projection $P$. We prove that the essential numerical range $W_{e}(\mathcal{T})$ of $\mathcal{T}$ can be described in terms of $W_{\infty}(\mathcal{T})$ as

$$
W_{e}(\mathcal{T})=\bigcup_{\mathcal{K} \in \mathcal{K}(H)^{n}} W_{\infty}(\mathcal{T}-\mathcal{K})
$$

Moreover there exists an $n$-tuple $\mathcal{K}$ of trace-class operators on $H$ such that $W_{e}(\mathcal{T})=\overline{W_{\infty}(\mathcal{T}-\mathcal{K})}$. We also show that for every tuple $\mathcal{T} \in B(H)^{n}$ of bounded linear operators on $H$ one has

$$
\overline{\operatorname{conv}} W(\mathcal{T})=\operatorname{conv}\left(W(\mathcal{T}) \cup W_{e}(\mathcal{T})\right),
$$

and

$$
\operatorname{Int} \overline{W(\mathcal{T})} \subset W(\mathcal{T})
$$

if $\operatorname{Int} W_{e}(T) \neq \emptyset$.

\section{Notation}

It will be convenient to fix some notations in a separate section. In particular, we let $H$ be a Hilbert space with the inner product $\langle\cdot, \cdot\rangle$, and $B(H)$ the space of all bounded linear operators on $H$. For a bounded linear operator $T$ we denote by $\sigma(T)$ its spectrum, and by $N(T)$ its kernel.

In the following we consider an $n$-tuple $\mathcal{T}=\left(T_{1}, \ldots, T_{n}\right) \in B(H)^{n}$. Note that we do not in general assume that the operators $T_{j}$ commute. For $x, y \in H$ we write shortly $\langle\mathcal{T} x, y\rangle=\left(\left\langle T_{1} x, y\right\rangle, \ldots,\left\langle T_{n} x, y\right\rangle\right) \in \mathbb{C}^{n}$ and $\mathcal{T} x=$ $\left(T_{1} x, \ldots, T_{n} x\right) \in H^{n}$. Similarly for $\lambda=\left(\lambda_{1}, \ldots, \lambda_{n}\right) \in \mathbb{C}^{n}$ we write $\mathcal{T}-\lambda=$ $\left(T_{1}-\lambda_{1}, \ldots, T-\lambda_{n}\right)$ and $\|\lambda\|=\max \left\{\left|\lambda_{1}\right|, \ldots,\left|\lambda_{n}\right|\right\}$. If $\mathcal{T}=\left(T_{1}, \ldots, T_{n}\right) \in$ $B(H)^{n}$ and $R, S \in B(H)$ then

$$
R \mathcal{T} S:=\left(R T_{1} S, \ldots, R T_{n} S\right) .
$$

Thus, in particular, if $\mathcal{T} \in B(H)^{n}$ and $P_{M}$ is the orthogonal projection from $H$ onto $M$ then $P_{M} \mathcal{T} P_{M}=\left(P_{M} T_{1} P_{M}, \ldots, P_{M} T_{n} P_{M}\right)$.

For a closed set $K \subset \mathbb{C}^{n}$ we denote by $\partial K$ the topological boundary of $K$, by Int $K$ the interior of $K$, by conv $K$ the convex hull of $K$, and by $\widehat{K}$ the polynomial hull of $K$. Recall that if $K \subset \mathbb{C}$ then $\widehat{K}$ is the union of $K$ with all bounded components of the complement $\mathbb{C} \backslash K$.

Finally, we let $\mathbb{T}$ stand for the unit circle $\{\lambda \in \mathbb{C}:|\lambda|=1\}, \mathbb{D}$ for the unit $\operatorname{disc}\{\lambda \in \mathbb{C}:|\lambda|<1\}$ and $\mathbb{R}_{+}=[0, \infty)$.

\section{Preliminaries}

We start with recalling certain basic notions and facts from the spectral theory of operator tuples on Hilbert spaces. They can be found e.g. in 24, Chapters 2-3]. 
Let $\mathcal{T}=\left(T_{1}, \ldots, T_{n}\right) \in B(H)^{n}$ be an $n$-tuple of commuting operators. Recall that its joint (Harte) spectrum $\sigma(\mathcal{T})$ can be defined as the complement of the set of those $\lambda=\left(\lambda_{1}, \ldots, \lambda_{n}\right) \in \mathbb{C}^{n}$ for which

$$
\sum_{j=1}^{n} L_{j}\left(T_{j}-\lambda_{j}\right)=\sum_{j=1}^{n}\left(T_{j}-\lambda_{j}\right) R_{j}=I
$$

for some $L_{j}, R_{j}, 1 \leq j \leq n$, from the algebra $B(H)$. There are two particularly useful subsets of $\sigma(\mathcal{T})$. The first one, the joint essential spectrum $\sigma_{e}(\mathcal{T})$ of $\mathcal{T}$, can be defined as the (Harte) spectrum of the $n$-tuple $\pi(\mathcal{T}):=$ $\left(\pi\left(T_{1}\right), \ldots, \pi\left(T_{n}\right)\right)$ in the Calkin algebra $B(H) / \mathcal{K}(H)$, where $\mathcal{K}(H)$ denotes the ideal of all compact operators on $H$, and $\pi: B(H) \rightarrow B(H) / \mathcal{K}(H)$ stands for the quotient map. The second one, the essential approximate spectrum $\sigma_{\pi e}(\mathcal{T})$ of $\mathcal{T}$ consists of all $\lambda=\left(\lambda_{1}, \ldots, \lambda_{n}\right) \in \mathbb{C}^{n}$ such that there exists an orthonormal sequence $\left(x_{k}\right)_{k \geq 1} \subset H$ satisfying

$$
\sum_{j=1}^{n}\left\|\left(T_{j}-\lambda_{j}\right) x\right\|=0 .
$$

It is easy to show that $\sigma_{\pi e}(\mathcal{T}) \subset \sigma_{e}(\mathcal{T})$. Note that if $n=1$ then $\sigma_{e}\left(T_{1}\right)=$ $\left\{\lambda_{1} \in \mathbb{C}: T_{1}-\lambda_{1}\right.$ is not Fredholm $\}$, and for $T \in B(H)$ and $\mathcal{T}=\left(T, T^{2}, \ldots, T^{n}\right) \in$ $B(H)^{n}$, one has $\sigma(\mathcal{T})=\left\{\left(\lambda, \ldots, \lambda^{n}\right): \lambda \in \sigma(T)\right\}$, where $\sigma$ can be replaced by either $\sigma_{e}$ or $\sigma_{\pi e}$. It is well-known that $\sigma(\mathcal{T})$ and $\sigma_{e}(\mathcal{T})$ are non-empty compact subsets of $\mathbb{C}^{n}$, while $\sigma_{\pi e}(\mathcal{T})$ can be empty even if $n=1$. Basic facts on essential spectra of operator tuples can be found in [24, Chapter III.19].

For not necessarily commuting $n$-tuple $\mathcal{T}$ denote by $\sigma_{p}(\mathcal{T})$ the point spectrum of $\mathcal{T}$, i.e., the set of all $n$-tuples $\lambda=\left(\lambda_{1}, \ldots, \lambda_{n}\right) \in \mathbb{C}^{n}$ such that $\bigcap_{j=1}^{n} N\left(T_{j}-\lambda_{j}\right) \neq\{0\}$. If $x \in \bigcap_{j=1}^{n} N\left(T_{j}-\lambda_{j}\right)$ then we will write $\mathcal{T} x=\lambda x$. Remark, however, that in fact we will not need a somewhat cumbersome spectral theory of non-commuting operator tuples.

As in the case of a single operator, it is often useful to relate $\sigma(\mathcal{T})$ to a larger and more easily computable set $W(\mathcal{T}) \subset \mathbb{C}^{n}$ called the joint numerical range of $\mathcal{T}$ and defined as

$$
W(\mathcal{T})=\left\{\left(\left\langle T_{1} x, x\right\rangle, \ldots,\left\langle T_{n} x, x\right\rangle\right): x \in H,\|x\|=1\right\} .
$$

The set $W(\mathcal{T})$ can be identified with a subset of $\mathbb{R}^{2 n}$ if one identifies the $n$-tuple $\mathcal{T}$ with the $2 n$-tuple $\left(\operatorname{Re} T_{1}, \operatorname{Im} T_{1}, \ldots, \operatorname{Re} T_{n}, \operatorname{Im} T_{n}\right)$ of selfadjoint operators. Unfortunately, if $n>1$, then $W(\mathcal{T})$ is not in general convex, see e.g. 21.

As in the spectral theory, there is also a notion of the joint essential numerical range $W_{e}(\mathcal{T})$ associated to $\mathcal{T}$. For $\mathcal{T}=\left(T_{1}, \ldots, T_{n}\right) \in B(H)^{n}$ we define $W_{e}(\mathcal{T})$ as the set of all $n$-tuples $\lambda=\left(\lambda_{1}, \ldots, \lambda_{n}\right) \in \mathbb{C}^{n}$ such that there exists an orthonormal sequence $\left(x_{k}\right)_{k \geq 1} \subset H$ with

$$
\lim _{k \rightarrow \infty}\left\langle T_{j} x_{k}, x_{k}\right\rangle=\lambda_{j}, \quad j=1, \ldots, n .
$$


Alternatively, $W_{e}(\mathcal{T})$ can be defined as

$$
W_{e}(\mathcal{T}):=\bigcap \overline{W\left(T_{1}+K_{1}, \ldots, T_{n}+K_{n}\right)}
$$

where the intersection is taken over all $n$-tuples $K_{1}, \ldots, K_{n}$ of compact operators on $H$. Recall that $W_{e}(\mathcal{T})$ is a nonempty, compact and, in contrast to $W(\mathcal{T})$, convex subset of $\overline{W(\mathcal{T})}$, see [5] or [21]. Note that as a straightforward consequence of the definitions above, if the $n$-tuple $\mathcal{T} \in B(H)^{n}$ is commuting then $\sigma_{\pi e}(\mathcal{T}) \subset W_{e}(\mathcal{T})$. Then the convexity of $W_{e}(\mathcal{T})$ implies that conv $\sigma_{e}(T) \subset W_{e}(T)$, see the proof of Corollary 4.3 below.

There's also a useful and related notion of the numerical range for tuples of elements of a unital Banach algebra $\mathcal{A}$. For $a=\left(a_{1}, \ldots, a_{n}\right) \in \mathcal{A}^{n}$ define

$$
V(a, \mathcal{A}):=\left\{\left(f\left(a_{1}\right), \ldots, f\left(a_{n}\right)\right): f \in \mathcal{A}^{*}, f(1)=\|f\|=1\right\},
$$

and recall that $f \in A^{*}$ such that $f(1)=\|f\|=1$ are called states. With such a definition, one has

$$
\overline{\operatorname{conv}} W(\mathcal{T})=V(\mathcal{T}, B(H))
$$

and

$$
W_{e}(\mathcal{T})=V(\pi(\mathcal{T}), B(H) / \mathcal{K}(H))
$$

The proofs of (3.2) and (3.3) can be found in 25, Theorem 1 and Theorem 2], respectively. For a comprehensive account of joint essential numerical ranges one may consult [21]. Very recently, several geometric properties of joint essential numerical ranges (as e.g. convexity) were extended in [18] to the setting of joint matricial essential ranges.

The next result due to Zenger is used in a number of operator-theoretical constructions. Its proof can be found e.g. in [6, p. 18-20].

Lemma 3.1 ((Zenger's Lemma)). Let $u_{1}, \ldots, u_{n} \in H$ be linearly independent, and let $\alpha_{1}, \ldots, \alpha_{n} \in \mathbb{R}_{+}$be such that $\sum_{k=1}^{n} \alpha_{k}=1$. Then there exist $w_{1}, \ldots, w_{n} \in \mathbb{C}$ and $u \in H,\|u\| \leq 1$, satisfying $\left\|\sum_{j=1}^{n} w_{j} u_{j}\right\| \leq 1$ and

$$
\left\langle w_{j} u_{j}, u\right\rangle=\alpha_{j}, \quad 1 \leq j \leq n .
$$

Note that $\left\langle\sum_{j=1}^{n} w_{j} u_{j}, u\right\rangle=1$, and so $u=\sum_{j=1}^{n} w_{j} u_{j}$.

\section{Spectra And numerical RAnges For tuples}

In the following we consider an $n$-tuple $\mathcal{T}=\left(T_{1}, \ldots, T_{n}\right) \in B(H)^{n}$. Note that we do not assume that the operators $T_{j}$ commute.

It was proved in [26, Corollary 4.2] that

$$
\text { Int } W_{e}(\mathcal{T}) \subset W(\mathcal{T}) \text {. }
$$

Moreover, if $\lambda=\left(\lambda_{1}, \ldots, \lambda_{n}\right) \in \operatorname{Int} W_{e}(\mathcal{T})$, then there exists an infinitedimensional subspace $L$ of $H$ such that

$$
P_{L} T_{j} P_{L}=\lambda_{j} P_{L}, \quad j=1, \ldots, n,
$$

where $P_{L}$ is the orthogonal projection on $L$. So, despite $T_{j}, 1 \leq j \leq n$, do not commute, in a number of situations of interest they have diagonal 
compressions to the same subspace. Moreover, (4.1) has important spectral consequences. It was shown in [26, Corollary 4.4] that

$$
\text { Int conv } \sigma_{e}(\mathcal{T}) \subset W(\mathcal{T}) .
$$

On the other hand, (4.1) has certain drawbacks. For instance, if one of the operators $T_{j}, 1 \leq j \leq n$, is compact then $\operatorname{Int} W_{e}\left(T_{1}, \ldots, T_{n}\right)=\emptyset$, and (4.1) says nothing. Thus, it is desirable, to obtain extensions of (4.1) shedding also light on $W(\mathcal{T})$ in the case of tuples with "small" essential numerical range. The next theorem serves just that purpose. Extending (4.1), it allows one to describe "big" subsets of $W(\mathcal{T})$ in spectral terms. The result is also related to [33, Theorem 2.2] where weaker statements have been obtained. As [33, Theorem 2.2], the theorem below depends on Zenger's Lemma, and also uses the following simple statement.

Lemma 4.1. Let $\mathcal{T} \in B(H)^{n}$. If $\lambda \in W_{e}(\mathcal{T})$, then for every $\delta>0$ and every subspace $M \subset H$ of finite codimension there exists a unit vector $x \in M$ such that $\|\langle\mathcal{T} x, x\rangle-\lambda\|<\delta$.

Proof. If $\lambda \in W_{e}(\mathcal{T})$ then there exists an orthonormal sequence $\left(x_{i}\right)_{i>1}$ in $H$ such that $\left\langle\mathcal{T} x_{i}, x_{i}\right\rangle \rightarrow \lambda, i \rightarrow \infty$. Let $M \subset H$ be a subspace of a finite codimension and $\delta>0$. We have $\left\|P_{M^{\perp}} x_{i}\right\| \rightarrow 0$, and so $\left\|P_{M} x_{i}-x_{i}\right\| \rightarrow 0$ as $i \rightarrow \infty$. Set

$$
u_{i}=\frac{P_{M} x_{i}}{\left\|P_{M} x_{i}\right\|}, \quad i \geq 1 .
$$

Then $\lim _{i \rightarrow \infty}\left\|u_{i}-x_{i}\right\|=0$ and $\lim _{i \rightarrow \infty}\left\langle\mathcal{T} u_{i}, u_{i}\right\rangle=\lambda$. Hence there exists $i_{0}$ such that $\left\|\left\langle\mathcal{T} u_{i_{0}}, u_{i_{0}}\right\rangle-\lambda\right\|<\delta$.

Theorem 4.2. Let $\mathcal{T}=\left(T_{1}, \ldots, T_{n}\right) \in B(H)^{n}$. Then

$$
\operatorname{Int} \operatorname{conv}\left(W_{e}(\mathcal{T}) \cup \sigma_{p}(\mathcal{T})\right) \subset W(\mathcal{T}) .
$$

Proof. Let $\lambda=\left(\lambda_{1}, \ldots, \lambda_{n}\right) \in \operatorname{Int} \operatorname{conv}\left(W_{e}(\mathcal{T}) \cup \sigma_{p}(\mathcal{T})\right)$. We show that $\lambda \in W(\mathcal{T})$. By considering the $n$-tuple $\left(T_{1}-\lambda_{1}, \ldots, T_{n}-\lambda_{n}\right)$ instead of $\left(T_{1}, \ldots, T_{n}\right)$, we can assume without loss of generality that $\left(\lambda_{1}, \ldots, \lambda_{n}\right)=$ $(0, \ldots, 0)$.

Let $r>0$ satisfy

$$
\left\{\left(\varepsilon_{1}, \ldots, \varepsilon_{n}\right): \max _{j}\left|\varepsilon_{j}\right| \leq r\right\} \subset \operatorname{conv}\left(W_{e}(T) \cup \sigma_{p}(T)\right) .
$$

Let $\lambda^{(1)}, \ldots, \lambda^{(m)} \in \sigma_{p}(\mathcal{T}) \backslash W_{e}(\mathcal{T})$ be a finite set such that

$$
\min \left\{\left\|\mu-\lambda^{(i)}\right\|: i=1, \ldots, m\right\}<\frac{r}{2}
$$

for all $\mu \in \sigma_{p}(\mathcal{T})$.

We show that

$$
\left\{\varepsilon \in \mathbb{C}^{n}:\|\varepsilon\| \leq \frac{r}{2}\right\} \subset \operatorname{conv}\left(W_{e}(\mathcal{T}) \cup\left\{\lambda^{(1)}, \ldots, \lambda^{(m)}\right\}\right) .
$$


Let $a_{1}, \ldots, a_{n} \in \mathbb{C}, \sum_{i=1}^{n}\left|a_{i}\right|=1$, and $c \in \mathbb{R}$. Assume that

$$
\operatorname{Re} \sum_{i=1}^{n} a_{i} z_{i} \geq c
$$

for all $z=\left(z_{1}, \ldots, z_{n}\right) \in W_{e}(\mathcal{T}) \cup\left\{\lambda^{(1)}, \ldots, \lambda^{(m)}\right\}$. Then

$$
\operatorname{Re} \sum_{i=1}^{n} a_{i} z_{i} \geq c-\frac{r}{2}
$$

for all $z=\left(z_{1}, \ldots, z_{n}\right) \in W_{e}(\mathcal{T}) \cup \sigma_{p}(\mathcal{T})$. Consequently,

$$
\operatorname{Re} \sum_{i=1}^{n} a_{i} z_{i} \geq c-\frac{r}{2} \quad \text { for all } \quad z \in \mathbb{C}^{n}, \quad\|z\| \leq r .
$$

Setting $z_{i}=e^{-i \arg a_{i}} r, 1 \leq i \leq n$, in the inequality above we infer that $c+\frac{r}{2} \leq 0$.

If $z \in \mathbb{C}^{n},\|z\| \leq \frac{r}{2}$, then

$$
\operatorname{Re} \sum_{i=1}^{n} a_{i} z_{i} \geq-\frac{r}{2} \geq c
$$

Since the convex hull of $W_{e}(\mathcal{T}) \cup\left\{\lambda^{(1)}, \ldots, \lambda^{(m)}\right\}$ is the intersection of all halfspaces containing it, this shows (4.4).

Fix now eigenvectors $u_{1}, \ldots, u_{m} \in H$ such that $\mathcal{T} u_{i}=\lambda^{(i)} u_{i},\left\|u_{i}\right\|=1$. Let

$$
F=\bigvee_{i=1}^{m} u_{i} \quad \text { and } \quad M=F^{\perp} \cap \bigcap_{j=1}^{n}\left(T_{j} F\right)^{\perp} \cap \bigcap_{j=1}^{n}\left(T_{j}^{*} F\right)^{\perp} .
$$

Clearly $\operatorname{dim} F<\infty$ and $\operatorname{codim} M<\infty$. Note that

$$
F \perp M, \quad T_{j} F \perp M \quad \text { and } \quad T_{j} M \perp F \quad \text { for all } j=1, \ldots, n .
$$

We construct a unit vector $x \in H$ satisfying $\left\langle T_{j} x, x\right\rangle=0$ for all $1 \leq j \leq n$ inductively as a limit of consecutive approximations. Set $x_{0}=v_{0}=w_{0}=0$. It will be convenient to separate the following fact.

Claim. Let $r>0, F, M \subset H$ be as above. Let $k \in \mathbb{N} \cup\{0\}$, let $v_{k} \in M$, $w_{k} \in F$ and $x_{k}=v_{k}+w_{k}$ satisfy

$$
\begin{gathered}
w_{k}=\sum_{i=1}^{m} t_{i} u_{i}, \quad\left\langle t_{i} u_{i}, w_{k}\right\rangle=\alpha_{i} \geq 0, \quad\left\|w_{k}\right\|^{2}=\sum_{i=1}^{m} \alpha_{i}, \\
\left\|x_{k}\right\|^{2}=1-2^{-k} \quad \text { and } \quad\left\|\left\langle\mathcal{T} x_{k}, x_{k}\right\rangle\right\|<\frac{r}{2^{k+2}} .
\end{gathered}
$$

Then there exist $v_{k+1}, w_{k+1}$ and $x_{k+1}$,

$$
x_{k+1}=v_{k+1}+w_{k+1}, \quad v_{k+1} \in M, w_{k+1} \in F,
$$


such that

$$
\begin{gathered}
w_{k+1}=\sum_{i=1}^{m} s_{i} u_{i}, \quad\left\langle s_{i} u_{i}, w_{k+1}\right\rangle=\beta_{i} \geq 0, \quad\left\|w_{k+1}\right\|^{2}=\sum_{i=1}^{m} \beta_{i}, \\
\left\|x_{k+1}\right\|^{2}=1-2^{-k-1}, \quad\left\|v_{k+1}-v_{k}\right\|^{2} \leq \frac{1}{2^{k+1}}
\end{gathered}
$$

and

$$
\left\|\left\langle\mathcal{T} x_{k+1}, x_{k+1}\right\rangle\right\|<\frac{r}{2^{k+3}}
$$

To prove the claim, let $\varepsilon=\left\langle\mathcal{T} x_{k}, x_{k}\right\rangle$. Since $\|\varepsilon\|<\frac{r}{2^{k+2}}$, there exist elements $\lambda^{(m+1)}, \ldots, \lambda^{\left(m^{\prime}\right)} \in W_{e}(\mathcal{T})$ and numbers $c_{1}, \ldots, c_{m^{\prime}} \geq 0$ such that $\sum_{i=1}^{m^{\prime}} c_{i}=1$ and

$$
\sum_{i=1}^{m^{\prime}} c_{i} \lambda^{(i)}=-\varepsilon 2^{k+1}
$$

By Zenger's Lemma, there are complex numbers $s_{1}, \ldots, s_{n}$ such that $w_{k+1}:=$ $\sum_{i=1}^{m} s_{i} u_{i}$ satisfies

$$
\left\langle s_{i} u_{i}, w_{k+1}\right\rangle=\alpha_{i}+\frac{c_{i}}{2^{k+1}} \quad \text { and } \quad\left\|w_{k+1}\right\|^{2}=\sum_{i=1}^{m}\left(\alpha_{i}+\frac{c_{i}}{2^{k+1}}\right) .
$$

Thus

$$
\left\langle\mathcal{T} w_{k+1}, w_{k+1}\right\rangle=\sum_{i=1}^{m}\left(\alpha_{i}+\frac{c_{i}}{2^{k+1}}\right) \lambda^{(i)} .
$$

The elements $\lambda^{(m+1)}, \ldots, \lambda^{\left(m^{\prime}\right)}$ belong to $W_{e}(\mathcal{T})$. Using Lemma 4.1 and the induction argument, we can construct unit vectors $y_{m+1}, \ldots, y_{m^{\prime}}$ in the following way.

Suppose that $m \leq s<m^{\prime}$ and that the vectors $y_{m+1}, \ldots, y_{s}$ have already been constructed. Set

$$
\begin{aligned}
G & :=\bigvee\left\{v_{k}, T_{j} v_{k}, y_{i}, T_{j} y_{i}: m+1 \leq i \leq s, 1 \leq j \leq n\right\}, \\
L & :=G^{\perp} \cap \bigcap_{j=1}^{n}\left(T_{j}^{*} G\right)^{\perp} .
\end{aligned}
$$

Then $\operatorname{dim} G<\infty$ and $\operatorname{codim} L<\infty$. Hence there exists a unit vector $y_{s+1} \in L \cap M$ such that

$$
\left\|\left\langle T y_{s+1}, y_{s+1}\right\rangle-\lambda^{(s+1)}\right\|<\frac{r}{4}
$$

If the vectors $y_{m+1}, \ldots, y_{m^{\prime}}$ are constructed, set

$$
v_{k+1}=v_{k}+\sum_{i=m+1}^{m^{\prime}}\left(\frac{c_{i}}{2^{k+1}}\right)^{1 / 2} y_{i} \quad \text { and } \quad x_{k+1}=v_{k+1}+w_{k+1} .
$$


Then

$$
\begin{array}{r}
\left\|v_{k+1}\right\|^{2}=\left\|v_{k}\right\|^{2}+\sum_{i=m+1}^{m^{\prime}} \frac{c_{i}}{2^{k+1}}, \\
\left\|v_{k+1}-v_{k}\right\|^{2}=\sum_{i=m+1}^{m^{\prime}} \frac{c_{i}}{2^{k+1}} \leq \frac{1}{2^{k+1}}
\end{array}
$$

and

$$
\begin{aligned}
\left\|x_{k+1}\right\|^{2} & =\left\|v_{k+1}\right\|^{2}+\left\|w_{k+1}\right\|^{2}=\left\|v_{k}\right\|^{2}+\sum_{i=m+1}^{m^{\prime}} \frac{c_{i}}{2^{k+1}}+\sum_{i=1}^{m}\left(\alpha_{i}+\frac{c_{i}}{2^{k+1}}\right) \\
& =\left\|v_{k}\right\|^{2}+\frac{1}{2^{k+1}}+\left\|w_{k}\right\|^{2}=\left\|x_{k}\right\|^{2}+\frac{1}{2^{k+1}}=1-\frac{1}{2^{k+1}} .
\end{aligned}
$$

Finally,

$$
\begin{aligned}
\left\|\left\langle\mathcal{T} x_{k+1}, x_{k+1}\right\rangle\right\| & =\left\|\left\langle\mathcal{T} v_{k}, v_{k}\right\rangle+\sum_{i=m+1}^{m^{\prime}} \frac{c_{i}}{2^{k+1}}\left\langle\mathcal{T} y_{i}, y_{i}\right\rangle+\left\langle\mathcal{T} w_{k+1}, w_{k+1}\right\rangle\right\| \\
& \leq\left\|\left\langle\mathcal{T} v_{k}, v_{k}\right\rangle+\sum_{i=m+1}^{m^{\prime}} \frac{c_{i} \lambda^{(i)}}{2^{k+1}}+\sum_{i=1}^{m}\left(\alpha_{i}+\frac{c_{i}}{2^{k+1}}\right) \lambda^{(i)}\right\| \\
& +\sum_{i=m+1}^{m^{\prime}} \frac{c_{i}}{2^{k+1}}\left\|\left\langle T y_{i}, y_{i}\right\rangle-\lambda^{(i)}\right\| \\
& \leq\left\|\left\langle T x_{k}, x_{k}\right\rangle+\frac{1}{2^{k+1}} \sum_{i=1}^{m^{\prime}} c_{i} \lambda^{(i)}\right\|+\sum_{m+1}^{m^{\prime}} \frac{c_{i}}{2^{k+1}} \cdot \frac{r}{4} \\
& =\frac{r}{2^{k+3}} .
\end{aligned}
$$

This finishes the proof of the claim.

Now construct the vectors $v_{k}, w_{k}$ and $x_{k}=v_{k}+w_{k}, k \in \mathbb{N}$, inductively as described in the Claim. Clearly $\left(v_{k}\right)_{k \geq 1}$ is a Cauchy sequence, and we let $v \in M$ be its limit. The sequence $\left(w_{k}\right)_{k \geq 1}$ is a bounded sequence in the finite-dimensional space $F$. By passing to a subsequence if necessary, we may assume that $\left(w_{k}\right)_{k \geq 1}$ is convergent, $w_{k} \rightarrow w \in F, k \rightarrow \infty$. The vector

$$
x=v+w=\lim _{k \rightarrow \infty}\left(v_{k}+w_{k}\right)=\lim _{k \rightarrow \infty} x_{k}
$$

satisfies $\|x\|=1$ and $\langle\mathcal{T} x, x\rangle=0$. This finishes the proof.

Theorem 4.2 yields the following generalization of [26, Corollary 4.4], replacing $\sigma_{e}(\mathcal{T})$ by $\sigma(\mathcal{T})$ there, cf. (4.3). The generalization complements [33. Corollary 2.3] where, for a commuting tuple $\mathcal{T} \in B(H)^{n}$, it was shown that

$$
\operatorname{conv} \sigma(\mathcal{T}) \subset \overline{W(\mathcal{T})}
$$


(Note that, as it will be clear below, we will be interested in spectral inclusions for the numerical range $W(\mathcal{T})$, rather than for its closure.)

Corollary 4.3. Let $\mathcal{T}=\left(T_{1}, \ldots, T_{n}\right) \in B(H)^{n}$ be a commuting n-tuple. Then

$$
\text { Int } \operatorname{conv} \sigma(\mathcal{T}) \subset W(\mathcal{T}) .
$$

Proof. First note that by [24, Corollary 19.16], the polynomial hulls $\widehat{\sigma}_{e}(\mathcal{T})$ and $\widehat{\sigma}_{\pi e}(\mathcal{T})$ coincide, so conv $\sigma_{e}(\mathcal{T})=\operatorname{conv} \sigma_{\pi e}(\mathcal{T})$. In view of convexity of $W_{e}(\mathcal{T})$, it follows that $\operatorname{conv} \sigma_{e}(\mathcal{T}) \subset W_{e}(\mathcal{T})$, and thus, in particular, $\widehat{\sigma}_{e}(\mathcal{T}) \subset W_{e}(\mathcal{T})$. Moreover, by [24, Theorem 19.18], the set $\sigma(\mathcal{T}) \backslash \widehat{\sigma}_{e}(\mathcal{T})$ consists of isolated eigenvalues of $\mathcal{T}$. Therefore, we have

$$
\operatorname{conv} \sigma(\mathcal{T})=\operatorname{conv}\left(\widehat{\sigma}_{e}(\mathcal{T}) \cup \sigma_{p}(\mathcal{T})\right) \subset \operatorname{conv}\left(W_{e}(\mathcal{T}) \cup \sigma_{p}(\mathcal{T})\right)
$$

The statement follows then from Theorem 4.2.

Statements like Theorem 4.2 specified for tuples $\left(T, T^{2}, \ldots, T^{n}\right) \in B(H)^{n}$ allow one to find appropriate tuples of powers of complex numbers in their joint numerical ranges $W\left(T, T^{2}, \ldots, T^{n}\right)$, thus revealing certain geometric properties of the orbits of $T$. For instance, the fact that $(0, \ldots, 0) \in$ $W\left(T, T^{2}, \ldots, T^{n}\right)$ yields an element $x \in H$ such that $x \perp T^{k} x$ for all $k$ between 1 and $n$. The latter property was introduced and characterized in spectral terms for unitary $T$ by Arveson, [2]. For its generalizations see [26]. In general, the structure of $W\left(T, T^{2}, \ldots, T^{n}\right)$ can be rather complicated even if $H$ is finite-dimensional, see e.g. [12.

The next theorem was proved in [26, Corollary 4.2 and Corollary 4.7]. It will be instrumental in Section 6 below dealing with asymptotic properties of compressions of powers.

Theorem 4.4. Let $T \in B(H)$ and let $\lambda$ belong to the interior of polynomial hull of $\sigma(T)$. Then

$$
\left(\lambda, \lambda^{2}, \ldots, \lambda^{n}\right) \in \operatorname{Int} W_{e}\left(T, T^{2}, \ldots, T^{n}\right) \subset W\left(T, T^{2}, \ldots, T^{n}\right) .
$$

for all $n \in \mathbb{N}$.

Note that the assumption on $\lambda$ as in the theorem above is quite natural and apparently close to optimal as the following statements show.

Proposition 4.5. Let $T \in B(H), n \in \mathbb{N}$. Suppose that

(i) $(0, \ldots, 0) \notin$ Int conv $W\left(T, T^{2}, \ldots, T^{n}\right)$;

(ii) there exists a unit vector $x \in H$ such that $x \perp T x, T^{2} x, \ldots, T^{2 n} x$.

Then $\sigma_{p}(T) \neq \emptyset$.

Proof. Suppose $x \in H$ satisfies (ii). Then, by (i), there exist complex numbers $c_{1}, \ldots, c_{n}$ such that

$$
\operatorname{Re} \sum_{j=1}^{n}\left\langle c_{j} T^{j} u, u\right\rangle \geq 0
$$


for all $u \in H$. Let $S=\sum_{j=1}^{n} c_{j} T^{j}$. Let $\alpha \in \mathbb{C}$ and $y=\alpha x+S x$. Then

$$
0 \leq \operatorname{Re}\langle S y, y\rangle=\operatorname{Re}\left\langle\alpha S x+S^{2} x, S x\right\rangle=\operatorname{Re}\left(\alpha\|S x\|^{2}+\left\langle S^{2} x, S x\right\rangle\right) .
$$

Since this is true for all $\alpha \in \mathbb{C}$, we have $S x=0$. So $0 \in \sigma_{p}(S)=$ $\sigma_{p}\left(\sum_{j=1}^{n} c_{j} T^{j}\right)=\left\{\sum_{j=1}^{n} c_{j} \lambda^{j}: \lambda \in \sigma_{p}(T)\right\}$ by the spectral mapping theorem for the point spectrum. Hence $\sigma_{p}(T) \neq \emptyset$.

Corollary 4.6. Let $T \in B(H), \sigma_{p}(T)=\emptyset$. Suppose that for all $n \in \mathbb{N}$ one has $(0, \ldots, 0) \in W\left(T, \ldots, T^{n}\right)$. Then

$$
(0, \ldots, 0) \in \operatorname{Int} \text { conv } W\left(T, \ldots, T^{n}\right)
$$

for all $n \in \mathbb{N}$.

\section{JOINT NUMERICAL RANGES REVISITED}

In this section we use the results proved above to provide alternative and, we believe, sometimes simpler proofs of the theorems describing essential and so-called infinite numerical ranges for tuples in terms of their compressions and higher rank numerical ranges. The results were (essentially) obtained in [21] and [22], see also [19], 28], and [31] for their single operator analogues, and 25] for complementary results. Moreover, our techniques allow us to prove several new results of independent interest.

Let for the rest of this section $H$ will stand for an infinite-dimensional separable Hilbert space. We start with general considerations on joint numerical ranges. Recall that the joint numerical range $W(\mathcal{T})$ is, in general, neither convex nor closed. Thus, it makes sense to describe the closed convex hull of $W(\mathcal{T})$ in terms of $W(\mathcal{T})$ and the related set $W_{e}(\mathcal{T})$. The following statement is an extension of a similar theorem due to Lancaster for single operators [17. Its proof is based on an idea of Williams from [32]. For a different, geometrical proof of the statement see [30] (and also [9, Theorem 2.1 and Corollary 2.3] for related results).

Theorem 5.1. Let $\mathcal{T}=\left(T_{1}, \ldots, T_{n}\right) \in B(H)^{n}$. Then

$$
\overline{\operatorname{conv}} W(\mathcal{T})=\operatorname{conv}\left(W(\mathcal{T}) \cup W_{e}(\mathcal{T})\right) .
$$

Proof. Since $W_{e}(\mathcal{T}) \subset \overline{W(\mathcal{T})}$, we have the inclusion " $\supset$ ".

Conversely, let $\left(\lambda_{1}, \ldots, \lambda_{n}\right) \in \overline{\operatorname{conv}} W(\mathcal{T})$. Recall that by (3.2) one has $\overline{\operatorname{conv}} W(\mathcal{T})=V(\mathcal{T}, B(H))$. So there exists a state $f \in B(H)^{*}$ such that $f\left(T_{j}\right)=\lambda_{j}$ for all $j=1, \ldots, n$. By Dixmier's theorem [13], one has a decomposition $f=\alpha f_{0}+(1-\alpha) f_{1}$, where $0 \leq \alpha \leq 1$ and $f_{0}, f_{1}$ are states on $B(H)$ such that $f_{0}$ annihilates the ideal of compact operators $\mathcal{K}(H)$ on $H$, and $f_{1}(A):=\operatorname{trace}(A S)$ for a fixed trace class operator $S \geq 0$ and all $A \in B(H)$. Hence there exist an orthonormal system $\left(e_{k}\right)_{k \geq 1} \subset H$ and positive numbers $\beta_{k}$ with $\sum_{k \geq 1} \beta_{k}=1$ such that $S=\sum_{k \geq 1} \beta_{k} e_{k} \otimes e_{k}$. Thus

$$
f_{1}(A)=\sum_{k \geq 1} \beta_{k}\left\langle A e_{k}, e_{k}\right\rangle
$$


for all $A \in B(H)$. Recall that a convex set in $\mathbb{C}^{n}$ is invariant with respect to taking infinite convex combinations of its elements (note that the set may be not closed), see e.g. [11] or [29]. Thus, since clearly $\left\{\left(T e_{k}, e_{k}\right): k \geq 1\right\} \subset$ conv $W(\mathcal{T})$, we have

$$
f_{1}(\mathcal{T}) \in \operatorname{conv} W(\mathcal{T})
$$

By (3.3), $V\left(\pi\left(T_{1}\right), \ldots, \pi\left(T_{n}\right), B(H) / \mathcal{K}(H)\right)=W_{e}(\mathcal{T})$, where $\pi: B(H) \rightarrow$ $B(H) / \mathcal{K}(H)$ is the quotient map. Hence $f_{0}(\mathcal{T}) \in W_{e}(\mathcal{T})$, and thus

$$
\left(\lambda_{1}, \ldots, \lambda_{n}\right)=\left(f\left(T_{1}\right), \ldots, f\left(T_{n}\right)\right) \in \operatorname{conv}\left(W(\mathcal{T}) \cup W_{e}(\mathcal{T})\right) .
$$

Despite the properties of joint numerical ranges are much more involved than the properties of numerical ranges for single operators, joint numerical ranges can be described in terms of other numerical ranges that are somewhat simpler to deal with. Let us recall now the definition of higher rank numerical ranges.

Definition 5.2. Let $\mathcal{T}=\left(T_{1}, \ldots, T_{n}\right) \in B(H)^{n}$. Let $k \in \mathbb{N} \cup\{\infty\}$. Define the $k$-th rank numerical range of $\mathcal{T}$ as the set of all $\lambda=\left(\lambda_{1}, \ldots, \lambda_{n}\right) \in \mathbb{C}^{n}$ such that there exists a subspace $L \subset H$ with $\operatorname{dim} L=k$ satisfying

$$
P_{L} T_{j} P_{L}=\lambda_{j} P_{L}, \quad j=1, \ldots, n .
$$

The set $W_{\infty}(\mathcal{T})$ is called the infinite numerical range of $\mathcal{T}$.

Clearly $W_{1}(\mathcal{T})$ is the usual joint numerical range and

$$
W_{1}(\mathcal{T}) \supset W_{2}(\mathcal{T}) \supset \cdots \supset W_{\infty}(\mathcal{T}) .
$$

It is easy to see that $W_{\infty}(\mathcal{T})$ can be empty even for $n=1$. (Consider an injective compact operator $T_{1}$.) Using [26, Corollary 4.2] and the definition of $W_{e}(\mathcal{T})$ it follows that

$$
\operatorname{Int}\left(W_{e}(\mathcal{T})\right) \subset W_{\infty}(\mathcal{T}) \subset W_{e}(\mathcal{T})
$$

for any $\mathcal{T} \in B(H)^{n}$. So $W_{\infty}(\mathcal{T})$ is large whenever $W_{e}(\mathcal{T})$ is large. On the other hand, in infinite-dimensional spaces the $k$-th rank numerical range is always nonempty for each $k \in \mathbb{N}$, as the following proposition (implicit in [22]) shows.

Proposition 5.3. Let $\mathcal{T}=\left(T_{1}, \ldots, T_{n}\right) \in B(H)^{n}$. Then $W_{k}(\mathcal{T}) \neq \emptyset$ for all $k \in \mathbb{N}$.

Proof. We prove the statement by induction on $n$. By e.g. [20, Theorem 1], one infers that $W_{k}\left(T_{1}\right) \neq \emptyset$ for any operator $T_{1} \in B(K)$ with $\operatorname{dim} K \geq 3 k-2$. In particular, $W_{k}\left(T_{1}\right) \neq \emptyset$ for all $k \in \mathbb{N}$. Suppose the statement is true for some $n-1 \geq 1$. Let $k \in \mathbb{N}$. By the induction assumption, $W_{4 k}\left(T_{1}, \ldots, T_{n-1}\right) \neq \emptyset$. So there exists $\left(\lambda_{1}, \ldots, \lambda_{n-1}\right) \in \mathbb{C}^{n-1}$ and a subspace $L \subset H$ with $\operatorname{dim} L=k$ such that

$$
P_{L} T_{j} P_{L}=\lambda_{j} P_{L}, \quad j=1, \ldots, n-1 .
$$


By the same result from [20], $W_{k}\left(P_{L} T_{n} P_{L}\right) \neq \emptyset$. So there exists $\lambda_{n} \in \mathbb{C}$ and a subspace $L^{\prime} \subset L$ with $\operatorname{dim} L^{\prime}=k$ and $P_{L^{\prime}} T_{n} P_{L^{\prime}}=\lambda_{n} P_{L^{\prime}}$. Hence $P_{L^{\prime}} T_{i} P_{L^{\prime}}=\lambda_{i} P_{L^{\prime}}, 1 \leq i \leq n$, so that $\left(\lambda_{1}, \ldots, \lambda_{n}\right) \in W_{k}\left(T_{1}, \ldots, T_{n}\right)$ and $W_{k}\left(T_{1}, \ldots, T_{n}\right) \neq \emptyset$.

For $k<\infty$ the higher rank numerical ranges $W_{k}(\mathcal{T})$ are, in general, not convex. However, they are always star-shaped, as we prove below. See [22, Proposition 4.1] for an analogous statement.

Theorem 5.4. Let $\mathcal{T}=\left(T_{1}, \ldots, T_{n}\right) \in B(H)^{n}$. Then for every $k \in \mathbb{N}$ the set $W_{k}(\mathcal{T})$ is star-shaped with star centers taken from $W_{m}(\mathcal{T})$ for any $m>k(2 n+1)$.

Proof. Let $k \in \mathbb{N}$. Fix $m>k(2 n+1)$. By Proposition 5.3, it follows that $W_{m}(\mathcal{T}) \neq \emptyset$, so we can choose $\lambda \in W_{m}(\mathcal{T}) \subset W_{k}(\mathcal{T})$. We show that $W_{k}(\mathcal{T})$ is star-shaped with the center $\lambda$.

Let $\mu \in W_{k}(\mathcal{T})$ and $t \in[0,1]$. There exists a subspace $L \subset H$ with $\operatorname{dim} L=k$ and $P_{L} \mathcal{T} P_{L}=\mu P_{L}$. Let $x_{1}, \ldots, x_{k}$ be an orthonormal set in $L$. Let $M \subset H$ satisfy $\operatorname{dim} M=m$ and $P_{M} \mathcal{T} P_{M}=\lambda P_{M}$. We construct an orthonormal set $y_{1}, \ldots, y_{k} \in M$ in the following way: Let $y_{1}$ be any unit vector in $M \cap\left\{L, T_{j} L, T_{j}^{*} L: 1 \leq j \leq n\right\}^{\perp}$. Choose inductively unit vectors $y_{s} \in M, 2 \leq s \leq k$, such that

$$
\begin{aligned}
& y_{s} \perp\left\{L, T_{j} L, T_{j}^{*} L: \quad 1 \leq j \leq n,\right\} \\
& y_{s} \perp\left\{y_{i}, T_{j} y_{i}, T_{j}^{*} y_{i}: \quad 1 \leq i \leq s-1,1 \leq j \leq n\right\} .
\end{aligned}
$$

Let

$$
u_{s}:=\sqrt{t} x_{s}+\sqrt{1-t} y_{s}, \quad s=1, \ldots, k \quad \text { and } \quad L^{\prime}:=\bigvee_{s=1}^{k} u_{s} .
$$

Clearly $\operatorname{dim} L^{\prime}=k$ and the vectors $u_{1}, \ldots, u_{k}$ form an orthonormal basis in $L^{\prime}$. If $y \in L^{\prime},\|y\|=1$, then $y=\sum_{s=1}^{k} \alpha_{s} u_{s}$ for some $\left\{\alpha_{s}: 1 \leq s \leq k\right\} \subset \mathbb{C}$ with $\sum_{s=1}^{k}\left|\alpha_{s}\right|^{2}=1$. We have

$$
\begin{aligned}
\langle\mathcal{T} y, y\rangle & =\left\langle\mathcal{T} \sum_{s=1}^{k} \alpha_{s} \sqrt{t} x_{s}, \sum_{s=1}^{k} \alpha_{s} \sqrt{t} x_{s}\right\rangle+2 \operatorname{Re}\left\langle\mathcal{T} \sum_{s=1}^{k} \alpha_{s} \sqrt{t} x_{s}, \sum_{s=1}^{k} \alpha_{s} \sqrt{1-t} y_{s}\right\rangle \\
& +\left\langle\mathcal{T} \sum_{s=1}^{k} \alpha_{s} \sqrt{1-t} y_{s}, \sum_{s=1}^{k} \alpha_{s} \sqrt{1-t} y_{s}\right\rangle \\
& =\mu\left\|\sum_{s=1}^{k} \alpha_{s} \sqrt{t} x_{s}\right\|^{2}+\lambda\left\|\sum_{s=1}^{k} \alpha_{s} \sqrt{1-t} x_{s}\right\|^{2} \\
& =t \mu+(1-t) \lambda .
\end{aligned}
$$

Hence $t \mu+(1-t) \lambda \in W_{k}(\mathcal{T})$, so the set $W_{k}(\mathcal{T})$ is star-shaped with the center at $\lambda \in W_{m}(\mathcal{T})$, as required.

Remark 5.5. It is easy to see that the closure $\overline{W_{k}(\mathcal{T})}$ is also star-shaped. 
The infinite and essential numerical range have "infinite-dimensional" nature. However it is possible to describe them in terms of "finite-dimensional" higher rank numerical ranges. Moreover, we characterize the infinite and essential numerical ranges of tuples by means of compressions of tuples to infinite-dimensional subspaces.

The equivalence (i) $\Leftrightarrow$ (ii) in the proposition below was fist proved in 22 , Theorem 4.1].

Proposition 5.6. Let $\mathcal{T}=\left(T_{1}, \ldots, T_{n}\right) \in B(H)^{n}$ and $\lambda=\left(\lambda_{1}, \ldots, \lambda_{n}\right) \in$ $\mathbb{C}^{n}$. The following statements are equivalent:

(i) $\lambda \in W_{\infty}(\mathcal{T})$;

(ii) $\lambda \in \bigcap_{k=1}^{\infty} W_{k}(\mathcal{T})$;

(iii) for every subspace $M \subset H$ of finite codimension there exists a unit vector $x \in M$ such that $\langle\mathcal{T} x, x\rangle=\lambda$.

Proof. The implication (i) $\Rightarrow$ (ii) is clear.

(ii) $\Rightarrow$ (iii): Let $M \subset H$ be a subspace of finite codimension. Let $k \in \mathbb{N}$, $k>\operatorname{codim} M$. By (ii), there exists a subspace $F \subset H$ with $\operatorname{dim} F=k$ and $P_{F} \mathcal{T} P_{F}=\lambda P_{F}$. Then $F \cap M \neq\{0\}$, and any unit vector in $F \cap M$ satisfies (iii).

(iii) $\Rightarrow$ (i): Using (iii), find a unit vector $x_{1} \in H$ such that $\left\langle\mathcal{T} x_{1}, x_{1}\right\rangle=\lambda$. Construct inductively a sequence $\left(x_{i}\right)_{i \geq 1} \subset H$ of unit vectors such that

$$
x_{i+1} \perp\left\{x_{m}, T_{j} x_{m}, T_{j}^{*} x_{m}: 1 \leq m \leq i, 1 \leq j \leq n\right\}
$$

and

$$
\left\langle\mathcal{T} x_{i}, x_{i}\right\rangle=\lambda
$$

for all $i \in \mathbb{N}$. Let $L=\bigvee_{i=1}^{\infty} x_{i}$. Clearly $L$ is an infinite-dimensional subspace with an orthonormal basis $\left(x_{i}\right)_{i \geq 1}$. Let $y \in L,\|y\|=1$, so that $y=\sum_{i=1}^{\infty} \alpha_{i} x_{i}$ where $\sum_{i=1}^{\infty}\left|\alpha_{i}\right|^{2}=1$. Then

$$
\langle\mathcal{T} y, y\rangle=\sum_{i=1}^{\infty}\left|\alpha_{i}\right|^{2}\left\langle\mathcal{T} x_{i}, x_{i}\right\rangle=\lambda \sum_{i=1}^{\infty}\left|\alpha_{i}\right|^{2}=\lambda,
$$

so that $P_{L} \mathcal{T} P_{L}=\mu P_{L}$.

Incidentally, in the general setting of operator tuples, Proposition 5.6 gives a partial answer to an old question of Fillmore, Stampfli and Pearcy 14, p. 190, Remark (4)] on the description for $T \in B(H)$ of the set of $\lambda \in \mathbb{C}$ such that $P(T-\lambda) P=0$ for an infinite-rank projection $P$. It can also be considered as a sharper version of [3, Theorem 3.1.1] where an approximate version of the proposition has been proved.

The equivalence (i) $\Leftrightarrow$ (ii) in the next result has been first obtained in 22 , Corollary 4.5].

Proposition 5.7. Let $\mathcal{T}=\left(T_{1}, \ldots, T_{n}\right) \in B(H)^{n}$ and $\lambda=\left(\lambda_{1}, \ldots, \lambda_{n}\right) \in$ $\mathbb{C}^{n}$. The following statements are equivalent:

(i) $\lambda \in W_{e}(\mathcal{T})$; 
(ii) $\lambda \in \bigcap_{k=1}^{\infty} \overline{W_{k}(\mathcal{T})}$;

(iii) for every $\delta>0$ and every subspace $M \subset H$ of finite codimension there exists a unit vector $x \in M$ such that $\|\langle\mathcal{T} x, x\rangle-\lambda\|<\delta$.

Proof. (iii) $\Rightarrow$ (i) is clear.

(ii) $\Rightarrow$ (iii): Let $M \subset H$ be a subspace of a finite codimension and $\delta>0$. Let $k \in \mathbb{N}, k>\operatorname{codim} M$. By (ii), there exists $\mu \in W_{k}(\mathcal{T})$ such that $\|\lambda-\mu\|<\delta$. Let $F$ be a subspace of $H$ with $\operatorname{dim} F=k$ and $P_{F} \mathcal{T} P_{F}=\mu P_{F}$. Then $F \cap M \neq\{0\}$. Let $x \in F \cap M$ be any unit vector. Then

$$
\|\langle\mathcal{T} x, x\rangle-\lambda\|=\|\mu-\lambda\|<\delta .
$$

(i) $\Rightarrow$ (iii): This is proved in Lemma 4.1

(iii) $\Rightarrow$ (ii): Let $\lambda \in \mathbb{C}^{n}$ satisfy (iii) for some $\delta>0$. Let $k \in \mathbb{N}$ be fixed. Choose inductively an orthonormal sequence $\left(x_{i}\right)_{i \geq 1} \subset H$ such that

$$
x_{i+1} \perp\left\{x_{m}, T_{j} x_{m}, T_{j}^{*} x_{m}: 1 \leq m \leq i, 1 \leq j \leq n\right\}
$$

and

$$
\left\|\left\langle\mathcal{T} x_{i+1}, x_{i+1}\right\rangle-\lambda\right\|<\delta
$$

Let $L=\bigvee_{i=1}^{\infty} x_{i}$. Then $\operatorname{dim} L=\infty$ and $\|\langle\mathcal{T} y, y\rangle-\lambda\|<\delta$ for all $y \in L$, $\|y\|=1$. Hence

$$
\left\|P_{L} T_{j} P_{L}-\lambda_{j} P_{L}\right\|<2 \delta, \quad j=1, \ldots, n .
$$

Note that $W_{k}\left(P_{L} \mathcal{T} P_{L}\right) \neq \emptyset$, and let $\mu \in W_{k}\left(P_{L} \mathcal{T} P_{L}\right)$. Then $\|\mu-\lambda\|<2 \delta$. Since $\delta>0$ was arbitrary, $\lambda \in \overline{W_{k}(\mathcal{T})}$.

One important application of Propositions 5.6, (iii) and 5.7, (iii) is the proof of convexity for $W_{\infty}(\mathcal{T})$ and $W_{e}(\mathcal{T})$. By different arguments, the convexity of $W_{e}(\mathcal{T})$ was first proved in [5, Lemma 3.1] (see also [21, Theorem $3.1]$ ), while the fact that $W_{\infty}(\mathcal{T})$ is convex was discovered in [22, Theorem $4.2]$, see also [28].

Theorem 5.8. Let $\mathcal{T}=\left(T_{1}, \ldots, T_{n}\right) \in B(H)^{n}$. Then the sets $W_{\infty}(\mathcal{T})$ and $W_{e}(\mathcal{T})$ are convex.

Proof. Let $\lambda, \mu \in W_{\infty}(\mathcal{T})$ and $t \in[0,1]$. Let $M \subset H, \operatorname{codim} M<\infty$. By Proposition 5.6. there exists $x \in M,\|x\|=1$ and $\langle\mathcal{T} x, x\rangle=\lambda$. Similarly there exists a unit vector $y \in M \cap\left\{x, T_{j} x, T_{j}^{*} x: 1 \leq j \leq n\right\}^{\perp}$ such that $\langle\mathcal{T} y, y\rangle=\mu$. Let $u=\sqrt{t} x+\sqrt{1-t} y$. Clearly $u \in M,\|u\|=1$ and

$$
\langle\mathcal{T} u, u\rangle=t\langle\mathcal{T} x, x\rangle+(1-t)\langle\mathcal{T} y, y\rangle=t \lambda+(1-t) \mu .
$$

By Proposition [5.6 again, $t \lambda+(1-t) \mu \in W_{\infty}(\mathcal{T})$.

The convexity of $W_{e}(\mathcal{T})$ can be proved similarly using Proposition 5.7 instead of Proposition 5.6.

Clearly $W_{e}(\mathcal{T})$ is stable under compact perturbations. The behaviour of $W_{\infty}(\mathcal{T})$ under compact perturbations is described in Theorem 5.10 below. To prove it, we need the following result of independent interest. 
Proposition 5.9. Let $\mathcal{T}=\left(T_{1}, \ldots, T_{n}\right) \in B(H)^{n}$, and let $\Lambda \subset W_{e}(\mathcal{T})$ be a countable set. Then there exists an n-tuple $\mathcal{K}=\left(K_{1}, \ldots, K_{n}\right)$ of trace-class normal operators on $H$ such that

$$
\Lambda \subset W_{\infty}(\mathcal{T}-\mathcal{K})
$$

Proof. Let $\Lambda=\left\{\lambda_{1}, \lambda_{2}, \ldots\right\}$. Let $f: \mathbb{N} \rightarrow \mathbb{N} \times \mathbb{N}$ be a bijection. For $s \in \mathbb{N}$ write $f(s)=\left(f_{1}(s), f_{2}(s)\right)$. We construct inductively an orthonormal sequence $\left(e_{s}\right)_{s \geq 1} \subset H$ in the following way: Choose a unit vector $e_{1}$ arbitrarily, fix $s \geq 2$ and suppose that the vectors $e_{1}, \ldots, e_{s-1} \in H$ have already been constructed. Since $\lambda_{f_{1}(s)} \in W_{e}(\mathcal{T})$, there exists an orthonormal sequence $\left(x_{k}\right)_{k \geq 1} \subset H$ such that $\lim _{k \rightarrow \infty}\left\langle\mathcal{T} x_{k}, x_{k}\right\rangle=\lambda_{f_{1}(s)}$. Let

$$
F_{s}=\bigvee\left\{e_{i}, T_{j} e_{i}, T_{j}^{*} e_{i}: 1 \leq i \leq s-1,1 \leq j \leq n\right\} .
$$

Since $\operatorname{dim} F_{s}<\infty$, there exists $m \in \mathbb{N}$ such that

$$
\left\|P_{F_{s}} x_{m}\right\| \leq 2^{-s} \quad \text { and } \quad\left\|\left\langle\mathcal{T} x_{m}, x_{m}\right\rangle-\lambda_{f_{1}(s)}\right\| \leq 2^{-s} .
$$

Set $e_{s}=\frac{\left(I-P_{F_{s}}\right) x_{m}}{\left\|\left(I-P_{F_{s}}\right) x_{m}\right\|}$. Then $\left\|e_{s}\right\|=1$ and $e_{s} \perp F_{s}$. We also have

$$
\begin{aligned}
\left\|x_{m}-e_{s}\right\| & \leq\left\|x_{m}-\left(I-P_{F_{s}}\right) x_{m}\right\|+\left\|\left(I-P_{F_{s}}\right) x_{m}-\frac{\left(I-P_{F_{s}}\right) x_{m}}{\left\|\left(I-P_{F_{s}}\right) x_{m}\right\|}\right\| \\
& \leq\left\|P_{F_{s}} x_{m}\right\|+\left(1-\frac{1}{1-2^{-s}}\right) \\
& \leq 3 \cdot 2^{-s} .
\end{aligned}
$$

Moreover, if $\varepsilon_{s}=\left(\varepsilon_{s, 1}, \ldots, \varepsilon_{s, n}\right) \in \mathbb{C}^{n}$ is given by $\varepsilon_{s}=\left\langle\mathcal{T} e_{s}, e_{s}\right\rangle-\lambda_{f_{1}(s)}$ then

$$
\begin{aligned}
\left|\varepsilon_{s, j}\right| & =\left|\left\langle T_{j} e_{s}, e_{s}\right\rangle-\lambda_{f_{1}(s), j}\right| \\
& \leq\left|\left\langle T_{j}\left(e_{s}-x_{m}\right), e_{s}\right\rangle\right|+\left|\left\langle T_{j} x_{m}, e_{s}-x_{m}\right\rangle\right|+\left|\left\langle T_{j} x_{m}, x_{m}\right\rangle-\lambda_{f_{1}(s), j}\right| \\
& \leq 3 \cdot 2^{-s}\left\|T_{j}\right\|+3 \cdot 2^{-s}\left\|T_{j}\right\|+2^{-s}, \quad 1 \leq j \leq n .
\end{aligned}
$$

Now, for every $j=1, \ldots, n$ define $K_{j} \in B(H)$ by

$$
K_{j}=\sum_{s=1}^{\infty} \varepsilon_{s, j} e_{s} \otimes e_{s} .
$$

By construction, $K_{j}$ is a trace-class normal operator and $\left\langle\left(T_{j}-K_{j}\right) e_{s}, e_{s}\right\rangle=$ $\lambda_{f_{1}(s), j}$ for all $s \in \mathbb{N}$.

For $k \in \mathbb{N}$ let $L_{k}=\bigvee\left\{e_{s}: f_{1}(s)=k\right\}$. Clearly $\operatorname{dim} L_{k}=\infty$. If $f_{1}(s)=k$ then $\left\langle T_{j} e_{s}, e_{s}\right\rangle=\lambda_{k, j}$. Moreover, if $f_{1}(s)=k=f_{1}\left(s^{\prime}\right)$ and $s \neq s^{\prime}$ then $\left\langle T_{j} e_{s}, e_{s^{\prime}}\right\rangle=0=\left\langle T_{j} e_{s^{\prime}}, e_{s}\right\rangle$ for all $j=1, \ldots, n$. It is easy to see that this means that

$$
P_{L_{k}}(\mathcal{T}-\mathcal{K}) P_{L_{k}}=\lambda_{k} P_{L_{k}}
$$

Hence $\lambda_{k} \in W_{\infty}(\mathcal{T}-\mathcal{K})$. 
Using Proposition [5.9, we can now express $W_{e}(\mathcal{T})$ in terms of the infinite numerical ranges of compact perturbations of $W_{\infty}(\mathcal{T})$. The result below seems to be new even for single operators.

Theorem 5.10. Let $\mathcal{T} \in B(H)^{n}$. Then:

(i) $W_{e}(\mathcal{T})=\bigcup_{\mathcal{K} \in \mathcal{K}(H)^{n}} W_{\infty}(\mathcal{T}-\mathcal{K})$;

(ii) there exists an $n$-tuple $\mathcal{K}$ of compact operators such that $W_{e}(\mathcal{T})=$ $\overline{W_{\infty}(\mathcal{T}-\mathcal{K})}$.

Proof. To show (i), observe that $W_{e}(\mathcal{T})=W_{e}(\mathcal{T}-\mathcal{K})$ and $W_{\infty}(\mathcal{T}-K) \subset$ $W_{e}(\mathcal{T})$ for any $n$-tuple of compact operators $\mathcal{K}$. Hence we have the inclusion " $\supset$ ". The other inclusion is clear by Proposition 5.9.

To prove (ii), it suffices to apply Proposition 5.9 with $\Lambda$ being any dense countable set in $W_{e}(T)$ and to use once again that $W_{e}(\mathcal{T})$ is invariant under compact perturbations. Since

$$
W_{e}(\mathcal{T})=\bar{\Lambda} \subset \overline{W_{\infty}(\mathcal{T}-\mathcal{K})} \subset W_{e}(\mathcal{T})
$$

the assertion follows.

Theorem [5.10 is a counterpart of [25, Corollary 13] where it was proved that for any $\mathcal{T} \in B(H)^{n}$ there exists an $n$-tuple of compact operators $\mathcal{K}$ such that $W_{e}(T)=\overline{W(\mathcal{T}-\mathcal{K})}$. Note that the theorem provides a one more proof of convexity of $W_{e}(\mathcal{T})$ once the convexity of $W_{\infty}(\mathcal{T})$ is established.

The notion of the infinite numerical range allows us to prove an inclusion result for numerical ranges which complements Theorem 4.2 and partially generalizes (4.1). (Note however that its proof uses (4.1) essentially.) Let us first remark that if $V \subset \mathbb{C}$ is a convex set, then $\operatorname{Int}(\bar{V}) \subset V$. Indeed, let $\lambda \in \operatorname{Int} \bar{V}$. We show that $\lambda \in V$. Without loss of generality we can assume that $\lambda=0$. Since $0 \in \operatorname{Int} \bar{V}$, there exists $r>0$ such that $\{a \in \mathbb{C}:|a| \leq$ $r\} \subset \bar{V}$. In particular, $a_{0}:=r, a_{1}:=r \eta$ and $a_{2}:=r \eta^{2}$ are elements of $\bar{V}$, where $\eta=e^{2 \pi i / 3}$. Now if the elements $b_{0}, b_{1}$, and $b_{2}$ belong to $V$, and are sufficiently close to $a_{0}, a_{1}$ and $a_{2}$, respectively, then $0 \in \operatorname{conv}\left\{b_{0}, b_{1}, b_{2}\right\}$. Since $V$ is convex, we have $0 \in V$.

Thus by convexity of $W(T)$ for any $T \in B(H)$, we infer that

$$
\text { Int } \overline{W(T)} \subset W(T) .
$$

While for tuples $\mathcal{T} \in B(H)^{n}$ the set $W(\mathcal{T})$ is in general not convex, the property (5.2) nevertheless holds also for $\mathcal{T}$ if $\operatorname{Int} W_{e}(\mathcal{T}) \neq \emptyset$.

Theorem 5.11. Let $\mathcal{T}=\left(T_{1}, \ldots, T_{n}\right) \in B(H)^{n}$, Int $\left(W_{e}(\mathcal{T})\right) \neq \emptyset$. Then Int $\overline{W(\mathcal{T})} \subset W(\mathcal{T})$.

Proof. Let $\operatorname{Int}\left(W_{e}(\mathcal{T}) \neq \emptyset\right.$. Without loss of generality we may assume that $(0, \ldots, 0) \in \operatorname{Int} W_{e}(\mathcal{T})$. Let $r>0$ satisfy

$$
\left\{\left(z=\left(z_{1}, \ldots, z_{n}\right):\|z\| \leq r\right\} \subset \operatorname{Int}\left(W_{e}(\mathcal{T})\right) .\right.
$$


Let $\lambda \in \operatorname{Int} \overline{W(\mathcal{T})}$, so there exists $s>0$ such that $(1+s) \lambda \in \operatorname{Int}(\overline{W(\mathcal{T})})$. Let $0<\delta<s r$. Then there is $\mu \in W(\mathcal{T})$ such that $\|\mu-(1+s) \lambda\|<\delta$. Set $\eta=\lambda+\frac{\lambda-\mu}{s}$. Then

$$
\|\eta\| \leq\left\|\lambda+\frac{\lambda-(1+s) \lambda}{s}\right\|+\frac{\delta}{s}<r
$$

So, by (15.1),$\eta \in \operatorname{Int}\left(W_{e}(\mathcal{T})\right) \subset W_{\infty}(\mathcal{T})$. Furthermore,

$$
\frac{1}{1+s} \mu+\frac{s}{1+s} \eta=\frac{1}{1+s} \mu+\frac{s}{1+s} \lambda+\frac{\lambda-\mu}{1+s}=\lambda .
$$

By Theorem 5.4, $\lambda \in W(\mathcal{T})$.

Since the interior of the essential numerical range played an important role above, it is natural to realize when it is non-empty. The following simple proposition clarifies the situation in algebraic terms. Let $\mathcal{S}$ stand for the real linear subspace of $B(H)$ formed by the sums of selfadjoint compact operators on $H$ and real scalar multiples of the identity.

Proposition 5.12. Let $\mathcal{T}=\left(T_{1}, \ldots, T_{n}\right) \in B(H)^{n}$. The following statements are equivalent:

(i) Int $W_{e}(\mathcal{T}) \neq \emptyset$;

ii) Int $W_{\infty}(\mathcal{T}) \neq \emptyset$;

(iii) the operators $\operatorname{Re} T_{1}, \operatorname{Im} T_{1}, \ldots, \operatorname{Re} T_{n}, \operatorname{Im} T_{n}$ are linearly independent in the real vector space of all selfadjoint operators modulo $\mathcal{S}$.

More precisely, if $c, t_{1}, \ldots, t_{2 n}$ are real numbers such that $\sum_{j=1}^{n}\left(t_{2 j-1} \operatorname{Re} T_{j}+\right.$ $\left.t_{2 j} \operatorname{Im} T_{j}\right)-c I$ is compact, then $t_{1}=\cdots=t_{2 n}=0$.

Proof. The equivalence (i) $\Leftrightarrow$ (ii) was proved above. Thus, it is enough to prove the equivalence (i) $\Leftrightarrow$ (iii). Recall that $W_{e}(\mathcal{T})$ is a nonempty convex set. We consider $W_{e}(\mathcal{T})$ to be a subset of $\mathbb{R}^{2 n}$. So Int $W_{e}(\mathcal{T})=\emptyset$ if and only if $W_{e}(\mathcal{T})$ is contained in a proper hyperplane in $\mathbb{R}^{2 n}$. This is equivalent to the existence of a non-trivial $(2 n)$-tuple $\left(t_{1}, \ldots, t_{2 n}\right) \in \mathbb{R}^{2 n}$ and $c \in \mathbb{R}$ such that

$$
\sum_{j=1}^{2 n} t_{j} z_{j}-c=0
$$

for all $\left(z_{1}, \ldots, z_{2 n}\right) \in W_{e}(\mathcal{T}) \subset \mathbb{R}^{2 n}$. This means that

$$
W_{e}\left(\sum_{j=1}^{n}\left(t_{2 j-1} \operatorname{Re} T_{j}+t_{2 j} \operatorname{Im} T_{j}\right)-c I\right) \subset\{0\},
$$

i.e.,

$$
\sum_{j=1}^{n}\left(t_{2 j-1} \operatorname{Re} T_{j}+t_{2 j} \operatorname{Im} T_{j}\right)-c I
$$

is a compact operator. 


\section{Asymptotics of COMPREssions FOR OPERATOR ITERATES}

In this section the numerical ranges ideology will be used to study asymptotical properties of powers of bounded operators. We will show that if the powers of $T \in B(H)$ vanish in the weak operator topology and the spectrum of $T$ is large enough, then for any strict contraction $C$ it is possible to find a subspace $L \subset H$ such that the compressions of $\left(T^{n}\right)_{L}$ to $L$ match asymptotically the powers of $C$ in the uniform operator topology. Moreover, if the assumption $T^{n} \rightarrow 0$ in the weak operator topology is dropped then for each $k \in \mathbb{N}$ we are able to construct a subspace $L \subset H$ such that $\left(T^{n}\right)_{L}=C_{u}^{n}, 1 \leq n \leq k$, where $C_{u} \in B(L)$ is a contraction unitarily equivalent to $C$.

In the rest of this section we fix a separable infinite-dimensional Hilbert space $H$. We first recall several additional notions and notations for operator tuples needed for the sequel. Let $\mathcal{A}_{j} \in B(H)^{n}, 1 \leq j \leq r$, so that $\mathcal{A}_{j}=$ $\left(A_{j 1}, \ldots, A_{j n}\right)$ for every $j$. The direct sum $\bigoplus_{j=1}^{r} \mathcal{A}_{j}$ is then defined as the $n$-tuple

$$
\left(\bigoplus_{j=1}^{r} A_{j 1}, \ldots, \bigoplus_{j=1}^{r} A_{j n}\right) \in B\left(\bigoplus_{j=1}^{r} H\right)^{n} .
$$

Note that if $M$ is a subspace of a Hilbert space $H$, and $T_{M}: M \rightarrow M$ is the compression $P_{M} T P_{M}$ of $T \in B(H)$ to $M$, then $T_{M}=J_{M}^{*} T J_{M}$, where the natural embedding $J_{M}: M \rightarrow H$ is defined by $J x=x, x \in M$. So, if $M \subset \bigoplus_{j=1}^{r} H$ and $\mathcal{A}_{j} \in B(H)^{n}, 1 \leq j \leq r$, then we define the compression $\left(\bigoplus_{j=1}^{r} \mathcal{A}_{j}\right)_{M}$ as the $n$-tuple

$$
J_{M}^{*}\left(\bigoplus_{j=1}^{r} \mathcal{A}_{j}\right) J_{M}=\left(J_{M}^{*}\left(\bigoplus_{j=1}^{r} A_{j 1}\right) J_{M}, \ldots, J_{M}^{*}\left(\bigoplus_{j=1}^{r} A_{j n}\right) J_{M}\right) \in B(M)^{n} .
$$

The next statement, of interest in itself, is an extension of [27, Proposition 1.1] from the case of a single operator to the case of operator tuples. It will allow us to identify a convex combination of operator tuples with a compression of their direct sum.

Lemma 6.1. Let $n, r \in \mathbb{N}$, and let $\mathcal{A}_{j} \in B(H)^{n}, j=1, \ldots, r$. Let $\alpha_{1}, \ldots, \alpha_{r} \geq 0, \sum_{j=1}^{r} \alpha_{j}=1$. Then there exists a subspace $M \subset \bigoplus_{j=1}^{r} H$ such that

$$
\left(\bigoplus_{j=1}^{r} \mathcal{A}_{j}\right)_{M} \stackrel{u}{\sim} \sum_{j=1}^{r} \alpha_{j} \mathcal{A}_{j}
$$

Proof. For $r=1$ the statement is trivial. We prove the statement first for $r=2$.

Consider the operator $U: H \oplus H \rightarrow H \oplus H$ defined by

$$
U=\left(\begin{array}{cc}
\sqrt{\alpha_{1}} & \sqrt{\alpha_{2}} \\
\sqrt{\alpha_{2}} & -\sqrt{\alpha_{1}}
\end{array}\right)
$$


It is easy to verify that $U^{*}=U=U^{-1}$. Moreover,

$$
U\left(\begin{array}{cc}
\mathcal{A}_{1} & 0 \\
0 & \mathcal{A}_{2}
\end{array}\right) U=\left(\begin{array}{cc}
\alpha_{1} \mathcal{A}_{1}+\alpha_{2} \mathcal{A}_{2} & * \\
* & *
\end{array}\right) .
$$

Let $M=U(H \oplus\{0\})$. Let $J_{M}: M \rightarrow H \oplus H$ be the natural embedding. Then

$$
J_{M} J_{M}^{*}=P_{M}=U\left(\begin{array}{ll}
1 & 0 \\
0 & 0
\end{array}\right) U,
$$

where $P_{M}$ is the orthogonal projection onto $M$. Let $J_{1}: H \rightarrow H \oplus H$ be defined by $J_{1} h=h \oplus 0, \quad h \in H$. Then $J_{M}^{*} U J_{1}: H \rightarrow M$ is a unitary operator.

We have

$$
\begin{aligned}
\left(\begin{array}{cc}
\mathcal{A}_{1} & 0 \\
0 & \mathcal{A}_{2}
\end{array}\right)_{M} & =J_{M}^{*}\left(\begin{array}{cc}
\mathcal{A}_{1} & 0 \\
0 & \mathcal{A}_{2}
\end{array}\right) J_{M} \\
& \stackrel{u}{\sim}\left(J_{1}^{*} U J_{M}\right) J_{M}^{*}\left(\begin{array}{cc}
\mathcal{A}_{1} & 0 \\
0 & \mathcal{A}_{2}
\end{array}\right) J_{M}\left(J_{M}^{*} U J_{1}\right) \\
& =J_{1}^{*} U P_{M}\left(\begin{array}{cc}
\mathcal{A}_{1} & 0 \\
0 & \mathcal{A}_{2}
\end{array}\right) P_{M} U J_{1} \\
& =J_{1}^{*}\left(\begin{array}{cc}
1 & 0 \\
0 & 0
\end{array}\right) U\left(\begin{array}{cc}
\mathcal{A}_{1} & 0 \\
0 & \mathcal{A}_{2}
\end{array}\right) U\left(\begin{array}{cc}
1 & 0 \\
0 & 0
\end{array}\right) J_{1} \\
& =J_{1}^{*}\left(\begin{array}{cc}
1 & 0 \\
0 & 0
\end{array}\right)\left(\begin{array}{cc}
\alpha_{1} \mathcal{A}_{1}+\alpha_{2} \mathcal{A}_{2} & * \\
& *
\end{array}\right)\left(\begin{array}{cc}
1 & 0 \\
0 & 0
\end{array}\right) J_{1} \\
& =J_{1}^{*}\left(\begin{array}{cc}
\alpha_{1} \mathcal{A}_{1}+\alpha_{2} \mathcal{A}_{2} & 0 \\
0 & 0
\end{array}\right) J_{1} \\
& =\alpha_{1} \mathcal{A}_{1}+\alpha_{2} \mathcal{A}_{2} .
\end{aligned}
$$

For $r>2$ the statement can be proved by induction. Let $r \geq 3$ be fixed and suppose the statement is true for $r-1$. We may assume that $\sum_{j=1}^{r-1} \alpha_{j} \neq$ 0 . By the induction hypothesis, there exists a subspace $L \subset \bigoplus_{j=1}^{r-1} H$ such that

$$
J_{L}^{*}\left(\bigoplus_{j=1}^{r-1} \mathcal{A}_{j}\right) J_{L} \stackrel{u}{\sim}\left(\sum_{j=1}^{r-1} \alpha_{j}\right)^{-1} \sum_{j=1}^{r-1} \alpha_{j} \mathcal{A}_{j}
$$

Consider the Hilbert space $L \oplus H$. By the statement for $r=2$, there exists a subspace $M \subset L \oplus H \subset \bigoplus_{j=1}^{r} H$ such that

$$
J_{M}^{*}\left(\bigoplus_{j=1}^{r} \mathcal{A}_{j}\right) J_{M} \stackrel{u}{\sim}\left(\sum_{j=1}^{r-1} \alpha_{j}\right) \cdot \frac{\sum_{j=1}^{r-1} \alpha_{j} \mathcal{A}_{j}}{\sum_{j=1}^{r-1} \alpha_{j}}+\alpha_{r} \mathcal{A}_{r}=\sum_{j=1}^{r} \alpha_{j} \mathcal{A}_{j}
$$

and the statement is thus true for $r$. This completes the proof.

Remark 6.2. Let $\mathcal{A}_{j} \in B(H)^{n}$ and $\alpha_{1}, \ldots, \alpha_{r}$ be as above. Let $\widetilde{\mathcal{A}}_{j} \in B(H)^{n}$ be $n$-tuples unitarily equivalent to $\mathcal{A}_{j}$, i.e., $\widetilde{\mathcal{A}}_{j}=U_{j}^{-1} \mathcal{A}_{j} U_{j}$ for some unitary operators $U_{j} \in B(H), j=1, \ldots, r$. 
Since $\bigoplus_{j=1}^{r} \mathcal{A}_{j} \stackrel{u}{\sim} \bigoplus_{j=1}^{r} \widetilde{\mathcal{A}}_{j}$, the previous lemma implies also that there exists a subspace $\widetilde{L} \subset H$ such that

$$
\left(\bigoplus_{j=1}^{r} \mathcal{A}_{j}\right)_{\widetilde{L}} \stackrel{u}{\sim} \sum_{j=1}^{r} \alpha_{j} \widetilde{\mathcal{A}}_{j} .
$$

Let $S \subset \mathbb{C}^{n}$. Denote by $\mathcal{M}(S)$ the set of all $n$-tuples of operators $\mathcal{A}=$ $\left(A_{1}, \ldots, A_{n}\right) \in B(H)^{n}$ such that there exist an orthonormal basis $\left(x_{i}\right)_{i \geq 1}$ in $H$ and elements $\lambda_{i} \in S, i \geq 1$, satisfying $\mathcal{A} x_{i}=\lambda_{i} x_{i}, i \geq 1$.

Using Proposition 6.1 we will further identify a compression of a tuple $\mathcal{T}$ with a tuple of diagonal operators $\mathcal{A}$ whose diagonals belong to the infinite numerical range of $\mathcal{T}$.

Proposition 6.3. Let $\mathcal{T}=\left(T_{1}, \ldots, T_{n}\right) \in B(H)^{n}$. Let $\mathcal{A} \in \operatorname{conv} \mathcal{M}\left(W_{\infty}(\mathcal{T})\right)$. Then there exists a subspace $L \subset H$ such that the compression $\mathcal{T}_{L}$ is unitarily equivalent to $\mathcal{A}$.

Proof. By assumption there exists $r \in \mathbb{N}$ such that

$$
\mathcal{A}=\sum_{j=1}^{r} \alpha_{j} \mathcal{A}_{j}
$$

for some $\mathcal{A}_{j} \in \mathcal{M}\left(W_{\infty}(\mathcal{T})\right), 1 \leq j \leq r$, and $\left(\alpha_{j}\right)_{1 \leq j \leq k}$ are nonnegative numbers satisfying $\sum_{j=1}^{r} \alpha_{j}=1$. So for every $j, 1 \leq j \leq r$, we have $\mathcal{A}_{j}=\operatorname{diag}\left(\lambda_{j, 1}, \lambda_{j, 2}, \ldots\right)$ with $\left(\lambda_{j, i}\right)_{i \geq 1} \in W_{\infty}(\mathcal{T})$.

Consider the set $\{1, \ldots, r\} \times \mathbb{N}$ with the lexicographic order: $(j, i) \prec\left(j^{\prime}, i^{\prime}\right)$ if either $i<i^{\prime}$ or $i=i^{\prime}$ and $j<j^{\prime}$. We construct inductively an orthonormal sequence $\left(x_{j, i}\right) \subset H, i, j \in \mathbb{N}$, in the following way: Fix a unit vector $x_{1,1} \in$ $H$, let $j \in\{1, \ldots, r\}, i \in \mathbb{N}$ and suppose that we have already constructed vectors $x_{j^{\prime}, i^{\prime}}$ for all $\left(j^{\prime}, i^{\prime}\right) \prec(j, i)$. Since $\lambda_{j, i} \in W_{\infty}(\mathcal{T})$, we can find a unit vector $x_{j, i} \in H$ such that

$$
\begin{array}{rlrl}
x_{j, i} \perp x_{j^{\prime}, i^{\prime}}, & \left(j^{\prime}, i^{\prime}\right) & \prec(j, i), & \\
x_{j, i} \perp T_{s} x_{j^{\prime}, i^{\prime}}, & \left(j^{\prime}, i^{\prime}\right) & \prec(j, i), s=1, \ldots, k, \\
x_{j, i} \perp T_{s}^{*} x_{j^{\prime}, i^{\prime}}, & \left(j^{\prime}, i^{\prime}\right) & \prec(j, i), s=1, \ldots, k,
\end{array}
$$

and

$$
\left\langle\mathcal{T} x_{j, i}, x_{j, i}\right\rangle=\lambda_{j, i}
$$

Suppose we have constructed the vectors $x_{j, i}$ in this way. For $j=1, \ldots, r$ let $H_{j}=\bigvee_{i \in \mathbb{N}} x_{j, i}$. Let $\widetilde{H}=\bigoplus_{j=1}^{r} H_{j}$. For $j=1, \ldots, r$ we have

$$
J_{H_{j}}^{*} \mathcal{T} J_{H_{j}} \stackrel{u}{\sim} \mathcal{A}_{j}
$$

and

$$
J_{\widetilde{H}}^{*} \mathcal{T} J_{\widetilde{H}} \stackrel{u}{\sim} \bigoplus_{j=1}^{r} \mathcal{A}_{j} .
$$


By Lemma 6.1, there exists a subspace $L \subset \widetilde{H} \subset H$ such that

$$
J_{L}^{*} \mathcal{T} J_{L} \stackrel{u}{\sim} \sum_{j=1}^{r} \alpha_{j} \mathcal{A}_{j}
$$

Now we are able to prove a partial generalization of [7, Theorem 2.1] by putting the result into the setting of operator tuples.

Theorem 6.4. Let $T \in B(H)$ be such that the polynomial hull $\hat{\sigma}(T)$ contains $\mathbb{D}$. Let $n \in \mathbb{N}$ and $\mathcal{T}=\left(T, T^{2}, \ldots, T^{n}\right)$. Let $A \in B(H),\|A\|<1$ and let $\mathcal{A}=\left(A, A^{2}, \ldots, A^{n}\right)$. Then there exists a subspace $L \subset H$ such that

$$
(\mathcal{T})_{L} \stackrel{u}{\sim} \mathcal{A}
$$

Proof. Let $\|A\|=c<1$. Since $A$ has the (power) dilation $c U$ where $U$ is a unitary operator, we can assume that $A=c U$.

By Theorem 4.4 and (5.1), we have $\left(\lambda, \lambda^{2}, \ldots, \lambda^{n}\right) \in W_{\infty}(\mathcal{T})$ for all $\lambda \in \mathbb{D}$. Since the function

$$
\lambda \mapsto \operatorname{dist}\left\{\left(\lambda, \ldots, \lambda^{n}\right), \mathbb{C}^{n} \backslash W_{\infty}(\mathcal{T})\right\}
$$

is continuous, we can find $c^{\prime}$ such that $c<c^{\prime}<1$ and $\frac{c^{\prime}}{c}\left(\lambda, \lambda^{2}, \ldots, \lambda^{n}\right) \in$ $W_{\infty}(\mathcal{T})$ for all $\lambda$ with $|\lambda|=c$. Set

$$
\eta=\left(1-\frac{c}{c^{\prime}}\right)(2 k)^{-1}
$$

Let $\delta$ be such that $\left(\varepsilon_{1}, \ldots, \varepsilon_{n}\right) \in W_{e}(\mathcal{T})$ for all $\varepsilon_{1}, \ldots, \varepsilon_{n} \in \mathbb{C}$ with $\max _{j}\left|\varepsilon_{j}\right|<$ $\delta$. By the Weyl-von Neumann diagonalization theorem (see e.g. 10, Chapter 6.37-38]), we can decompose $A$ as $A=D+K_{1}$, where $D$ is a diagonal operator with entries of modulus $c$ and $K_{1}$ is a compact operator satisfying $\left\|K_{1}\right\|<\delta \eta k^{-1}$. Set $K_{j}:=A^{j}-D^{j}, 1 \leq j \leq n$. We have

$$
K_{j}=A^{j}-D^{j}=\sum_{i=0}^{j-1} A^{i}(A-D) D^{j-i-1} .
$$

So for every $j$ the operator $K_{j}$ is compact and

$$
\left\|K_{j}\right\| \leq n c^{j-1}\|A-D\| \leq n\left\|K_{1}\right\| \leq \delta \eta
$$

Write $K_{j}=\operatorname{Re} K_{j}+i \operatorname{Im} K_{j}, 1 \leq j \leq n$. The operators $\operatorname{Re} K_{j}$ and $i \operatorname{Im} K_{j}$ are diagonal operators with entries of modulus at most $\delta \eta$. We have

$$
\begin{aligned}
\left(A, A^{2}, \ldots, A^{n}\right) & =\frac{c}{c^{\prime}} \cdot \frac{c^{\prime}}{c}\left(D, D^{2}, \ldots, D^{n}\right)+\eta \cdot \eta^{-1}\left(\operatorname{Re} K_{1}, 0, \ldots, 0\right) \\
& +\eta \cdot \eta^{-1}\left(i \operatorname{Im} K_{1}, 0, \ldots, 0\right)+\eta \cdot \eta^{-1}\left(0, \operatorname{Re} K_{2}, 0, \ldots, 0\right)+\cdots \\
& \cdots+\eta \cdot \eta^{-1}\left(0, \ldots, 0, i \operatorname{Im} K_{n}\right)
\end{aligned}
$$

where $\frac{c}{c^{\prime}}+2 k \eta=1$ and the $n$-tuples

$$
\frac{c^{\prime}}{c}\left(D, D^{2}, \ldots, D^{n}\right), \eta^{-1}\left(\operatorname{Re} K_{1}, 0, \ldots, 0\right), \ldots, \eta^{-1}\left(0, \ldots, 0, i \operatorname{Im} K_{n}\right)
$$


belong to $\mathcal{M}\left(W_{\infty}(\mathcal{T})\right)$. By Proposition 6.3, there exists a subspace $L \subset H$ such that

$$
(\mathcal{T})_{L} \stackrel{u}{\sim} \mathcal{A}
$$

We proceed with an asymptotic version of Theorem 6.4. To this aim several auxiliary lemmas will be needed. First we prove a slight generalization of [26, Lemma 6.1] where we replace the element $(0, \ldots, 0)$ by $\left(\lambda, \lambda^{2}, \ldots, \lambda^{n}\right),|\lambda|<1$, and choose a unit vector $x \in H$ more carefully. The next statement can be considered as a variant of the main result, Theorem 6.7 below, for a single vector.

Lemma 6.5. Let $T \in B(H)$ be such that $T^{n} \rightarrow 0$ in the weak operator topology, and let $\lambda \in \mathbb{C},|\lambda|<1$. Suppose that

$$
\left(\lambda, \lambda^{2}, \ldots, \lambda^{n}\right) \in W_{\infty}\left(T, \ldots, T^{n}\right)
$$

for all $n \in \mathbb{N}$. Let $A \subset H$ be a finite set, $\varepsilon>0$ and $M \subset H$ be a subspace of a finite codimension. Then there exists a unit vector $x \in M \cap A^{\perp}$ such that

$$
\sup _{n \geq 1}\left|\left\langle\left(T^{n}-\lambda^{n}\right) x, x\right\rangle\right| \leq \varepsilon, \quad \sup _{n \geq 1}\left|\left\langle T^{n} x, a\right\rangle\right| \leq \varepsilon \quad \text { and } \quad \sup _{n \geq 1}\left|\left\langle T^{* n} x, a\right\rangle\right| \leq \varepsilon
$$

for all $a \in A$.

Proof. Clearly $T$ is power bounded by the uniform boundedness principle. Let $K=\sup \left\{\left\|T^{n}\right\|: n=0,1, \ldots\right\}$. It is apparent that also $T^{* n} \rightarrow 0$ in the weak operator topology. Without loss of generality we may assume that $\max \{\|a\|: a \in A\} \leq 1$.

Choose $s \in \mathbb{N}$ such that $s>25 K^{2} \varepsilon^{-2}$, and find $n_{0} \in \mathbb{N}$ such that $|\lambda|^{n_{0}}<\frac{\varepsilon}{5}$.

We construct unit vectors $u_{1}, u_{2}, \ldots, u_{s} \in M$ and positive integers $n_{0}<$ $n_{1}<\cdots<n_{s}$ in the following way: Fix a unit vector $u_{1} \in M$, let $1 \leq$ $r \leq s-1$ and suppose that the unit vectors $u_{1}, \ldots, u_{r} \in M$ and numbers $n_{1}<\cdots<n_{r}$ have already been constructed.

By Proposition 5.7, there exists a unit vector $u_{r+1} \in M$ such that

$$
u_{r+1} \perp\left\{T^{n} u_{k}, T^{* n} u_{k}, T^{n} a, T^{* n} a: 0 \leq n \leq n_{r}, 1 \leq k \leq r, a \in A\right\}
$$

and

$$
\left\langle T^{n} u_{r+1}, u_{r+1}\right\rangle=\lambda^{n}, \quad 1 \leq n \leq n_{r} .
$$

Find $n_{r+1}>n_{r}$ such that

$$
\begin{aligned}
\left|\left\langle T^{n} u_{r+1}, u_{k}\right\rangle\right| & <\frac{\varepsilon}{5 s}, \\
\left|\left\langle T^{* n} u_{r+1}, u_{k}\right\rangle\right| & <\frac{\varepsilon}{5 s}, \\
\left|\left\langle T^{n} u_{r+1}, a\right\rangle\right| & <\frac{\varepsilon}{5 s},
\end{aligned}
$$

and

$$
\left|\left\langle T^{* n} u_{r+1}, a\right\rangle\right|<\frac{\varepsilon}{5 s}
$$

for all $n \geq n_{r+1}, 1 \leq k \leq r+1$ and $a \in A$. 
Let $u_{1}, \ldots, u_{s}$ and $n_{0}, \ldots, n_{s}$ be constructed in this way. Set

$$
x=\frac{1}{\sqrt{s}} \sum_{k=1}^{s} u_{k} .
$$

Clearly $x \in M$. Moreover, $\|x\|=1$ since the vectors $u_{k}$ are orthonormal.

For $n>n_{s}$ we have

$$
\left|\left\langle\left(T^{n}-\lambda^{n}\right) x, x\right\rangle\right| \leq s^{-1} \sum_{k, k^{\prime}=1}^{s}\left|\left\langle T^{n} u_{k}, u_{k^{\prime}}\right\rangle\right|+|\lambda|^{n} \leq s^{-1} s^{2} \frac{\varepsilon}{5 s}+\frac{\varepsilon}{5}<\varepsilon .
$$

Let $0 \leq r \leq s-1$ and $n_{r}<n \leq n_{r+1}$. Then

$$
\begin{aligned}
\left|\left\langle\left(T^{n}-\lambda^{n}\right) x, x\right\rangle\right| & \leq\left|\left\langle T^{n} x, x\right\rangle\right|+\frac{\varepsilon}{5} \\
& \leq s^{-1} \sum_{k, k^{\prime}=1}^{r}\left|\left\langle T^{n} u_{k}, u_{k^{\prime}}\right\rangle\right|+s^{-1} \sum_{k=1}^{r+1}\left|\left\langle T^{n} u_{r+1}, u_{k}\right\rangle\right| \\
& +s^{-1} \sum_{k=1}^{r}\left|\left\langle T^{n} u_{k}, u_{r+1}\right\rangle\right|+s^{-1} \sum_{k=r+2}^{s}\left|\left\langle T^{n} u_{k}, u_{k}\right\rangle\right| \\
& +s^{-1} \sum_{\substack{1 \leq k, k^{\prime} \leq s, k \neq k^{\prime} \\
\max \left\{k, k^{\prime}\right\} \geq r+2}}\left|\left\langle T^{n} u_{k}, u_{k^{\prime}}\right\rangle\right|+\frac{\varepsilon}{5},
\end{aligned}
$$

where the last sum is equal to 0 by the construction. So

$$
\begin{aligned}
\left|\left\langle\left(T^{n}-\lambda^{n}\right) x, x\right\rangle\right| & \leq s^{-1} r^{2} \frac{\varepsilon}{5 s}+s^{-1}\left\|T^{n} u_{r+1}\right\| \cdot\left\|\sum_{k=1}^{r+1} u_{k}\right\| \\
& +s^{-1}\left\|T^{* n} u_{r+1}\right\| \cdot\left\|\sum_{k=1}^{r} u_{k}\right\|+s^{-1}(s-r-1)|\lambda|^{n}+\frac{\varepsilon}{5} \\
& \leq \frac{\varepsilon}{5}+s^{-1} K \sqrt{r+1}+s^{-1} K \sqrt{r}+\frac{\varepsilon}{5}+\frac{\varepsilon}{5} \\
& \leq \varepsilon .
\end{aligned}
$$

Let $1 \leq n \leq n_{0}$. Then

$$
\begin{aligned}
& \left\langle\left(T^{n}-\lambda^{n}\right) x, x\right\rangle \\
= & s^{-1} \sum_{k=1}^{s}\left\langle\left(T^{n}-\lambda^{n}\right) u_{k}, u_{k}\right\rangle+s^{-1} \sum_{1 \leq k, k^{\prime} \leq s, k \neq k^{\prime}}\left\langle\left(T^{n}-\lambda^{n}\right) u_{k}, u_{k^{\prime}}\right\rangle=0 .
\end{aligned}
$$

Hence

$$
\sup _{n \geq 1}\left|\left\langle\left(T^{n}-\lambda^{n}\right) x, x\right\rangle\right| \leq \varepsilon .
$$

Let $a \in A$. For $n \geq n_{s}$ we have

$$
\left|\left\langle T^{n} x, a\right\rangle\right| \leq \frac{1}{\sqrt{s}} \sum_{k=1}^{s}\left|\left\langle T^{n} u_{k}, a\right\rangle\right| \leq \frac{1}{\sqrt{s}} \cdot s \cdot \frac{\varepsilon}{5 s}<\varepsilon .
$$


Let $0 \leq r \leq s-1$ and $n_{r} \leq n<n_{r+1}$. Then

$$
\begin{aligned}
\left|\left\langle T^{n} x, a\right\rangle\right| & \leq \frac{1}{\sqrt{s}} \sum_{k=1}^{r}\left|\left\langle T^{n} u_{k}, a\right\rangle\right|+\frac{1}{\sqrt{s}}\left|\left\langle T^{n} u_{r+1}, a\right\rangle\right|+\frac{1}{\sqrt{s}} \sum_{k=r+2}^{s}\left|\left\langle T^{n} u_{k}, a\right\rangle\right| \\
& \leq \frac{1}{\sqrt{s}} \cdot r \cdot \frac{\varepsilon}{5 s}+\frac{1}{\sqrt{s}} \cdot K+0<\varepsilon .
\end{aligned}
$$

Finally, for $1 \leq n \leq n_{0}$ we have

$$
\left\langle T^{n} x, a\right\rangle=s^{-1} \sum_{k=1}^{s}\left\langle T^{n} u_{k}, a\right\rangle=0 .
$$

Thus

$$
\sup _{n \geq 1}\left|\left\langle T^{n} x, a\right\rangle\right| \leq \varepsilon
$$

for all $a \in A$.

The property $\sup _{n \geq 1}\left|\left\langle T^{* n} x, a\right\rangle\right| \leq \varepsilon$ for all $a \in A$ can be proved similarly.

The previous lemma enables us to prove the main result in a particular situation when the compression of powers a bounded operator is approximated by powers of any strictly contractive diagonal operator.

Lemma 6.6. Let $T \in B(H)$ be such that $T^{n} \rightarrow 0$ in the weak operator topology and $\sigma(T) \supset \mathbb{T}$. Let $\left(\lambda_{k}\right)_{k \geq 1} \subset \mathbb{D}$, $\sup _{k \geq 1}\left|\lambda_{k}\right|<1$, and $\varepsilon>0$. Then there exists an orthonormal sequence $\left(e_{k}\right)_{k \geq 1}$ in $H$ such that

$$
\begin{gathered}
\sup _{n \geq 1}\left\|\left(T^{n}\right)_{L}-D^{n}\right\| \leq \varepsilon \\
\text { and } \lim _{n \rightarrow \infty}\left\|\left(T^{n}\right)_{L}-D^{n}\right\|=0,
\end{gathered}
$$

where $L=\bigvee_{k=1}^{\infty} e_{k}$ and $D \in B(L)$ is the diagonal operator defined by $D e_{k}=$ $\lambda_{k} e_{k}, \quad k \in \mathbb{N}$.

Proof. Let $r=\sup _{k}\left|\lambda_{k}\right|<1$. By Theorem 4.4 and (5.1),

$$
\left(\lambda_{k}, \lambda_{k}^{2}, \ldots, \lambda_{k}^{n}\right) \in W_{\infty}\left(T, T^{2}, \ldots, T^{n}\right)
$$

for all $n, k \in \mathbb{N}$. Let $n_{0} \in \mathbb{N}$ satisfy $r^{n_{0}}<\frac{\varepsilon}{16}$. We construct vectors $e_{1}, e_{2}, \ldots$ and positive integers $n_{1}<\cdots$ also inductively.

Fix a unit vector $e_{1} \in H$, suppose that $s \geq 1$ and orthonormal vectors $e_{1}, \ldots, e_{s} \in H$ and numbers $n_{0}<n_{1}<\cdots<n_{s}$ have already been constructed. Using Lemma 6.5, find a unit vector $e_{s+1} \in H$ such that

$$
\begin{aligned}
& e_{s+1} \perp\left\{T^{n} e_{k}, T^{* n} e_{k}: 0 \leq n \leq n_{s}, 1 \leq k \leq s\right\}, \\
& \sup _{n \geq 1}\left|\left\langle\left(T^{n}-\lambda_{s+1}^{n}\right) e_{s+1}, e_{s+1}\right\rangle\right|<\frac{\varepsilon}{2^{s+4}(s+1)}, \\
& \sup _{n \geq 1}\left|\left\langle T^{n} e_{s+1}, e_{k}\right\rangle\right|<\frac{\varepsilon}{2^{s+4}(s+1)}, \quad 1 \leq k \leq s,
\end{aligned}
$$


and

$$
\sup _{n \geq 1}\left|\left\langle T^{* n} e_{s+1}, e_{k}\right\rangle\right|<\frac{\varepsilon}{2^{s+4}(s+1)}, \quad 1 \leq k \leq s .
$$

Find $n_{s+1}>n_{s}$ such that $r^{n_{s+1}}<\frac{\varepsilon}{2^{s+5}}$ and

$$
\left|\left\langle T^{n} e_{k}, e_{k^{\prime}}\right\rangle\right| \leq \frac{\varepsilon}{2^{s+4}(s+1)}, \quad 1 \leq k, k^{\prime} \leq s+1, n \geq n_{s+1} .
$$

Let $L=\bigvee_{k=1}^{\infty} e_{k}$ and let the diagonal operator $D: L \rightarrow L$ be defined by $D e_{k}=\lambda_{k} e_{k}, \quad k \in \mathbb{N}$.

Let $x \in L,\|x\|=1$. Then $x=\sum_{k \geq 1} \alpha_{k} e_{k}$ where $\sum_{k \geq 1}\left|\alpha_{k}\right|^{2}=1$. Note that $\sum_{k=1}^{s}\left|\alpha_{k}\right| \leq \sqrt{s}$ for all $s \in \mathbb{N}$.

For $1 \leq n \leq n_{0}$ we have

$$
\begin{aligned}
\left|\left\langle\left(T^{n}-D^{n}\right) x, x\right\rangle\right| & \leq \sum_{k, k^{\prime}=1}^{\infty}\left|\alpha_{k} \bar{\alpha}_{k^{\prime}}\right| \cdot\left|\left\langle\left(T^{n}-D^{n}\right) e_{k}, e_{k^{\prime}}\right\rangle\right| \\
& =\sum_{k=1}^{\infty}\left|\alpha_{k}\right|^{2} \cdot\left|\left\langle\left(T^{n}-\lambda_{k}^{n}\right) e_{k}, e_{k}\right\rangle\right| \leq \frac{\varepsilon}{2} .
\end{aligned}
$$

Thus $\left\|\left(T^{n}\right)_{L}-D^{n}\right\| \leq \varepsilon$.

Let $s \geq 0$ and $n_{s}<n \leq n_{s+1}$. Then

$$
\begin{aligned}
\left|\left\langle\left(T^{n}-D^{n}\right) x, x\right\rangle\right| & \leq\left|\left\langle T^{n} x, x\right\rangle\right|+r^{n} \\
& \leq\left|\sum_{k, k^{\prime}=1}^{\infty} \alpha_{k} \bar{\alpha}_{k^{\prime}}\left\langle T^{n} e_{k}, e_{k^{\prime}}\right\rangle\right|+\frac{\varepsilon}{2^{s+4}} \\
& \leq \sum_{k, k^{\prime}=1}^{s}\left|\alpha_{k} \bar{\alpha}_{k^{\prime}}\right| \cdot\left|\left\langle T^{n} e_{k}, e_{k^{\prime}}\right\rangle\right|+\sum_{k=1}^{s}\left|\alpha_{s+1} \bar{\alpha}_{k}\right| \cdot\left|\left\langle T^{n} e_{s+1}, e_{k}\right\rangle\right| \\
& +\sum_{k=1}^{s}\left|\alpha_{k} \bar{\alpha}_{s+1}\right| \cdot\left|\left\langle T^{n} e_{k}, e_{s+1}\right\rangle\right|+\sum_{k=s+1}^{\infty}\left|\alpha_{k}\right|^{2} \cdot\left|\left\langle T^{n} e_{k}, e_{k}\right\rangle\right| \\
& +\sum_{k \neq k^{\prime}, \max \left\{k, k^{\prime}\right\} \geq s+2}\left|\alpha_{k} \bar{\alpha}_{k^{\prime}}\right| \cdot\left|\left\langle T^{n} e_{k}, e_{k^{\prime}}\right\rangle\right|+\frac{\varepsilon}{2^{s+4}} \\
& \leq \frac{s \varepsilon}{2^{s+3} s}+\frac{\varepsilon \sqrt{s}}{2^{s+4}(s+1)}+\frac{\varepsilon \sqrt{s}}{2^{s+4}(s+1)} \\
& +\sum_{k=s+1}^{\infty}\left|\alpha_{k}\right|^{2} \cdot\left(\left|\left\langle\left(T^{n}-D^{n}\right) e_{k}, e_{k}\right\rangle\right|+r^{n}\right)+0+\frac{\varepsilon}{2^{s+4}} \\
& \leq \frac{\varepsilon}{2^{s+3}}+\frac{\varepsilon}{2^{s+4}} \cdot \frac{\varepsilon}{2^{s+4}}+\left(\frac{\varepsilon}{2^{s+4}}+\frac{\varepsilon}{2^{s+4}}\right)+\frac{\varepsilon}{2^{s+4}} \\
& <
\end{aligned}
$$

Thus

$$
\sup \left\{\left|\left\langle\left(T^{n}-D^{n}\right) x, x\right\rangle\right|: x \in L,\|x\|=1\right\} \leq \frac{\varepsilon}{2^{s+1}}
$$

and so $\left\|\left(T^{n}\right)_{L}-D^{n}\right\| \leq \frac{\varepsilon}{2^{s}}$. 
Hence

$$
\sup _{n \geq 1}\left\|\left(T^{n}\right)_{L}-D^{n}\right\| \leq \varepsilon \quad \text { and } \quad \lim _{n \rightarrow \infty}\left\|\left(T^{n}\right)_{L}-D^{n}\right\|=0 .
$$

Now using dilation theory, we can replace a strictly contractive diagonal operator $D$ in Lemma 6.6 by any strict contraction unitarily equivalent to a given one.

Theorem 6.7. Let $T \in B(H)$ be such that $T^{n} \rightarrow 0$ in the weak operator topology, and let $\sigma(T) \supset \mathbb{T}$. Let $\tilde{C} \in B(H)$ and $\|\tilde{C}\|<1$. Then for every $\varepsilon>0$ there exists a subspace $L \subset H$ and $C \in B(L)$ unitarily equivalent to $\tilde{C}$ such that

$$
\sup _{n \geq 1}\left\|\left(T^{n}\right)_{L}-C^{n}\right\| \leq \varepsilon
$$

and

$$
\lim _{n \rightarrow \infty}\left\|\left(T^{n}\right)_{L}-C^{n}\right\|=0 .
$$

Proof. Let $\|\tilde{C}\|<c<1$. Then $\tilde{C}$ has the power dilation $c \tilde{U}$ on a Hilbert space $\tilde{K}$, where $\tilde{U} \in B(\tilde{K})$ is the bilateral shift of infinite multiplicity. So it is sufficient to show the statement for the operator $c \tilde{U}$.

Find $k \in \mathbb{N}$ such that $\sup _{n \geq 1} n c^{n}<\frac{k \varepsilon}{4 \pi}$. For $0 \leq j \leq k-1$ let $E_{j} \subset \tilde{K}$ be the spectral subspace of $\tilde{U}$ corresponding to the set $\left\{e^{2 \pi i t}: j / k \leq t<(j+\right.$ $1) / k\}$. Consider the operator $\tilde{D} \in B(\tilde{K})$ defined by $\tilde{D} x=e^{2 \pi i j / k} x, x \in E_{j}$. Then $\|\tilde{U}-\tilde{D}\| \leq \frac{2 \pi}{k}$ and similarly, $\left\|\tilde{U}^{n}-\tilde{D}^{n}\right\| \leq \frac{2 \pi n}{k}$ for all $k$ and $n$ from $\mathbb{N}$. Thus

$$
\sup _{n \geq 1}\left\|(c \tilde{U})^{n}-(c \tilde{D})^{n}\right\| \leq \sup _{n \geq 1} \frac{2 \pi n c^{n}}{k} \leq \varepsilon / 2
$$

and

$$
\lim _{n \rightarrow \infty}\left\|(c \tilde{U})^{n}-(c \tilde{D})^{n}\right\|=0 .
$$

Moreover, $c \tilde{D}$ is a diagonal operator of the form considered in Lemma 6.6. Hence, by Lemma 6.6, there exists a subspace $K \subset H$ and a unitarily equivalent copy $c D \in B(K)$ of $c \tilde{D}$ such that

$$
\sup _{n \geq 1}\left\|\left(T^{n}\right)_{K}-(c D)^{n}\right\| \leq \varepsilon / 2
$$

and

$$
\lim _{n \rightarrow \infty}\left\|\left(T^{n}\right)_{K}-(c D)^{n}\right\|=0
$$

Thus

$$
\sup _{n \geq 1}\left\|\left(T^{n}\right)_{K}-(c U)^{n}\right\| \leq \varepsilon
$$

and

$$
\lim _{n \rightarrow \infty}\left\|\left(T^{n}\right)_{K}-(c U)^{n}\right\|=0,
$$


where $U \in B(K)$ is unitarily equivalent to $\tilde{U}$. Hence there exits a subspace $L \subset K$ and a unitarily equivalent copy $C \in B(L)$ of $\tilde{C}$ such that

$$
\sup _{n \geq 1}\left\|\left(T^{n}\right)_{L}-C^{n}\right\| \leq \varepsilon \quad \text { and } \quad \lim _{n \rightarrow \infty}\left\|\left(T^{n}\right)_{L}-C^{n}\right\|=0 .
$$

\section{REFERENCES}

[1] J. H. Anderson and J. G. Stampfli, Commutators and compressions, Israel J. Math. 10 (1971), 433-441.

[2] W. Arveson, A theorem on the action of abelian unitary groups, Pacific J. Math. 16 (1966), 205-212.

[3] P. Binding, D. R. Farenick, and C.-K. Li, A dilation and norm in several variable operator theory, Canad. J. Math. 47 (1995), 449-461.

[4] H. Bercovici, C Foias, and A. Tannenbaum, The structured singular value for linear input/output operators, SIAM J. Control Optim. 34 (1996), 1392-1404.

[5] H. Bercovici, C. Foias, and C. Pearcy, Dilation theory and systems of simultaneous equations in the predual of an operator algebra, I, Michigan Math. J. 30 (1983), 335-354.

[6] F. Bonsall and J. Duncan, Complete Normed Algebras, Springer, Berlin, 1973.

[7] J.-C. Bourin, Compressions and pinchings, J. Operator Theory 50 (2003), 211-220.

[8] J.-C. Bourin and E.-Y. Lee, Pinchings and positive linear maps, J. Funct. Anal. 270 (2016), 359-374.

[9] J.-T. Chan, A note on the boundary of the joint numerical range, Linear and Multilinear Algebra 66 (2018), 821-826.

[10] J. B. Conway, A course in operator theory, Graduate Studies in Mathematics, 21, AMS,, Providence, RI, 2000.

[11] W. D. Cook and R. J. Webster, Carathéodory's theorem, Canad. Math. Bull. 15 (1972), 293.

[12] C. Davis, C.-K. Li, and A. Salemi, Polynomial numerical hulls of matrices, Linear Algebra Appl. 428 (2008), 137-153.

[13] J. Dixmier, Les fonctionelles lineáires sur lénsemble des operateurs bornes d'un espace de Hilbert, Ann. of Math. 51 (1950), 387-408.

[14] P. A. Fillmore, J. G. Stampfli, and J. P. Williams, On the essential numerical range, the essential spectrum, and a problem of Halmos, Acta Sci. Math. (Szeged) 33 (1972), 179-192.

[15] K. E. Gustafson and D. K. M. Rao, Numerical range, Universitext, Springer, New York, 1997.

[16] D. Hamdan, Fourier-Stieltjes transforms of Rajhman measures with full support on the circle, J. Dyn. Control Syst. 19 (2013), 277-286.

[17] J. Lancaster, The boundary of the numerical range, Proc. Amer. Math. Soc. 49 (1975), 393-398.

[18] P.-S. Lau, C.-K. Li, Y.-T. Poon, and N.-S. Sze, Convexity and star-shapedness of matricial range, J. Funct. Anal., in press, https://doi.org/10.1016/j.jfa.2018.03.018.

[19] C.-K. Li, Y.-T. Poon, N.-S. Sze, Higher rank numerical ranges and low rank perturbations of quantum channels, J. Math. Anal. Appl. 348 (2008), 843-855.

[20] C.-K. Li, Y.-T. Poon, N.-S. Sze, Condition for the higher rank numerical range to be non-empty, Linear and Multilinear Algebra 57 (2009), 365-368.

[21] C.-K. Li and Y.-T. Poon, The joint essential numerical range of operators: convexity and related results, Studia Math. 194 (2009), 91-104.

[22] C.-K. Li and Y.-T. Poon, Generalized numerical ranges and quantum error correction, J. Operator Theory 66 (2011), 335-351. 
[23] J. Loreaux and G. Weiss, Diagonality and idempotents with applications to problems in operator theory and frame theory, J. Operator Theory 75 (2016), 91-118.

[24] V. Müller, Spectral theory of linear operators and spectral systems in Banach algebras, Second ed., Operator Theory: Advances and Applications, 139, Birkhäuser, Basel, 2007.

[25] V. Müller, The joint essential numerical range, compact perturbations, and the Olsen problem, Studia Math. 197 (2010), 275-290.

[26] V. Müller and Yu. Tomilov, Circles in the spectrum and the geometry of orbits: a numerical ranges approach, J. Funct. Anal. 274 (2018), 433-460.

[27] A. Pokrzywa, Limits of spectra of strongly converging compressions, J. Operator Theory 12 (1984), 199-212.

[28] E. Roldán-Pensado, Infinite numerical range is convex, Linear and Multilinear Algebra, 56 (2008), 731-733.

[29] H. Rubin and O. Wesler, A note on convexity in Euclidean n-space, Proc. Amer. Math. Soc. 9(1958), 522-523.

[30] M. Takaguchi and M Cho, The joint numerical range and the joint essential numerical range, Sci Rep. Hirosaki Univ. 27 (1980), 6-8.

[31] H. Woerdeman, The higher rank numerical range is convex, Linear and Multilinear Algebra, 56 (2008), 65-67.

[32] J. P. Williams, The numerical range and the essential numerical range, Proc. Amer. Math. Soc 66 (1977), 185-186.

[33] V. Wrobel, Joint spectra and joint numerical ranges for pairwise commuting operators in Banach spaces, Glasgow Math. J. 30 (1988), 145-153.

Institute of Mathematics,, Czech Academy of Sciences,, Žitna Str. 25, 115 67 Prague, Czech Republic

E-mail address: muller@math.cas.cz

Institute of Mathematics,, Polish Academy of Sciences, Śniadeckich Str. 8, 00-956 Warsaw,, Poland

E-mail address: ytomilov@impan.pl 